\section{Social protection}

\author{
(Tables 10.2 to $10.11,10.13$ and 10.15 to 10.19 )
}

Tables 10.2 to $10.6,10.9$ to 10.11 and 10.13 to 10.19 give details of contributors and beneficiaries under the National Insurance and Industrial Injury Acts, supplementary benefits and war pensions.

There are four classes of National Insurance Contributions (NICS):

Class 1 Earnings-related contributions paid on earnings from employment. Employees pay primary Class 1 contributions and employers pay secondary Class 1 contributions. Payment of Class 1 contributions builds up entitlement to contributory benefits which include Basic State Pension; Additional State Pension (State Earnings Related Pension Scheme SERPS and from April 2002, State Second Pension, S2P); Contribution Based Jobseeker's Allowance; Bereavement Benefits; Incapacity Benefit and the new Employment and Support Allowance.

Primary class 1 contributions stop at State Pension age, but not Class 1 secondary contributions paid by employers. There are reduced contribution rates where the employee contracts out of S2P (previously SERPS). They still receive a Basic State Pension but an Occupational or Personal Pension instead of the Additional State Second Pension.

Class 2 Flat rate contributions paid by the self-employed whose profits are above the small earnings exception. Payment of Class 2 contributions builds up entitlement to the contributory benefits, which include Basic State Pension, Bereavement Benefits, Maternity Allowance and Incapacity Benefit and the Employment and Support Allowance, but not Additional State Second Pension or Contribution Based Jobseeker's Allowance (JSA).

Class 2 contributions stop at State Pension age.

Class 3 Flat rate voluntary contributions, which can be paid by someone whose contribution record is insufficient. Payment of Class 3 contributions builds up entitlement to contributory benefits which include Basic State Pension and Bereavement Benefits. (Tables 10.2 to $10.11,10.13$ and 10.15 to 10.19$)$ Tables 10.2 to $10.6,10.9$ to 10.11 and 10.13 to 10.19 give details of contributors and beneficiaries under the National Insurance and Industrial Injury Acts, supplementary benefits and war pensions.

Class 4 Profit-related contributions paid by the self employed in addition to Class 2 contributions. Class 4 contributions stop at State Pension age. Under some circumstances people who are not in employment do not have to make voluntary contributions to accrue a qualifying year for Basic State Pension.

Home Responsibilities Protection

Home Responsibilities Protection (HRP) helps to protect the basic State Pension of those precluded from regular employment because they are caring for children or a sick or disabled person at home. To be entitled to HRP, a person must have been precluded from regular employment for a full tax year. HRP reduces the amount of qualifying years a person would otherwise need for a Basic State Pension.

\section{National Insurance Credits}

In addition to paying, or being treated as having paid contributions, a person can be credited with National Insurance. Contribution credits help to protect people's rights to State Retirement Pension and other Social Security Benefits.

A person is likely to be entitled to contributions credits if they are: a student in full time education or training, in receipt of Jobseeker's Allowance, unable to work due to sickness or disability, entitled to Statutory Maternity Pay or Statutory Adoption Pay, or they have received Carer's Allowance.

Credits are automatically awarded for men aged 60 to 65 provided they are not liable to pay Class 1 or $2 \mathrm{NICs}$, and to young people for the tax years containing their 16th, 17th and 18th birthdays.

\section{Jobseeker's Allowance}

\section{(Table 10.6)}

Jobseeker's Allowance (JSA) replaced Unemployment Benefit and Income Support for unemployed claimants on 7 October 1996. It is a unified benefit with two routes of entry: contribution-based, which depends mainly upon National Insurance contributions, and income-based, which depends mainly upon a means test. Some claimants can qualify by either route. In practice they receive income-based JSA but have an underlying entitlement to the contribution based element. 


\section{Employment and support allowance, Invalidity Benefit and Incapacity Benefit}

\section{(Tables 10.7)}

Incapacity Benefit replaced Sickness Benefit and Invalidity Benefit from 13 April 1995. The first condition for entitlement to these contributory benefits is that the claimants are incapable of work because of illness or disablement. The second is that they satisfy the contribution conditions, which depend on contributions paid as an employed (Class 1) or self-employed person (Class 2). Under Sickness and Invalidity Benefits the contribution conditions were automatically treated as satisfied if a person was incapable of work because of an industrial accident or prescribed disease. Under Incapacity Benefit those who do not satisfy the contribution conditions do not have them treated as satisfied. Class 1A contributions paid by employers are in respect of the benefit of cars provided for the private use of employees, and the free fuel provided for private use. These contributions do not provide any type of benefit cover.

Since 6 April 1983, most people working for an employer and paying National Insurance contributions as employed persons receive Statutory Sick Pay (SSP) from their employer when they are off work sick. Until 5 April 1986 SSP was payable for a maximum of eight weeks, since this date SSP has been payable for 28 weeks. People who do not work for an employer, and employees who are excluded from the SSP scheme, or those who have run out of SSP before reaching the maximum of 28 weeks and are still sick, can claim benefit. Any period of SSP is excluded from the tables.

Spells of incapacity of three days or less do not count as periods of interruption of employment and are excluded from the tables. Exceptions are where people are receiving regular weekly treatment by dialysis or treatment by radiotherapy, chemotherapy or plasmapheresis where two days in any six consecutive days make up a period of interruption of employment, and those whose incapacity for work ends within three days of the end of SSP entitlement.

At the beginning of a period of incapacity, benefit is subject to three waiting days, except where there was an earlier spell of incapacity of more than three days in the previous eight weeks. Employees entitled to SSP for less than 28 weeks and who are still sick can get Sickness Benefit or Incapacity Benefit Short Term (Low) until they reach a total of 28 weeks provided they satisfy the conditions.

After 28 weeks of SSP and/or Sickness Benefit (SB), Invalidity Benefit (IVB) was payable up to pension age for as long as the incapacity lasted. From pension age, IVB was paid at the person's State Pension rate, until entitlement ceased when SP was paid, or until deemed pension age (70 for a man, 65 for a woman). People who were on Sickness or Invalidity Benefit on 12 April 1995 were automatically transferred to Incapacity Benefit, payable on the same basis as before.

For people on Incapacity Benefit under State Pension age there are two short-term rates: the lower rate is paid for the first 28 weeks of sickness and the higher rate for weeks 29 to 52 . From week 53 the Long Term rate Incapacity Benefit is payable. The Short Term rate Incapacity Benefit is based on State Pension entitlement for people over State Pension age and is paid for up to a year if incapacity began before pension age.

The long-term rate of Incapacity Benefit applies to people under State Pension age who have been sick for more than a year. People with a terminal illness, or who are receiving the higher rate care component of Disability Living Allowance, will get the Long Term rate. The Long Term rate is not paid for people over pension age.

Under Incapacity Benefit, for the first 28 weeks of incapacity, people previously in work will be assessed on the 'own occupation' test - the claimant's ability to do their own job. Otherwise, incapacity will be based on a personal capability assessment, which will assess ability to carry out a range of work-related activities. The test will apply after 28 weeks of incapacity or from the start of the claim for people who did not previously have a job. Certain people will be exempted from this test.

The tables exclude all men aged over 65 and women aged over 60 who are in receipt of State Pension, and all people over deemed pension age (70 for a man and 65 for a woman), members of the armed forces, mariners while at sea, and married women and certain widows who have chosen not to be insured for sickness benefit. The tables include a number of individuals who were unemployed prior to incapacity.

The Short Term (Higher) and Long Term rates of Incapacity Benefit are treated as taxable income. There were transitional provisions for people who were on Sickness or Invalidity Benefit on 12 April 1995. They were automatically transferred to Incapacity Benefit, payable on the same basis as before. Former IVB recipients continue to get Additional Pension entitlement, but frozen at 1994 levels. Also their IVB is not subject to tax. If they were over State Pension age on 12 April 1995 they may get Incapacity Benefit for up to five years beyond pension age. 


\section{Employment and Support Allowance}

Employment and Support Allowance (ESA) replaced Incapacity Benefit and Income Support paid on the grounds of incapacity for new claims from 27 October 2008. ESA consists of two phases. The first, the assessment phase rate, is paid for the first 13 weeks of the claim whilst a decision is made on the claimants capability through the 'Work Capability Asessment'. The second, or main phase begins after 14 weeks, but only if the 'Work Capability Assesment' has deemed the claimants illness or disability as a limitation on their ability to work.

Within the main phase there are two groups, 'The Work Related Activity Group' and 'The Support Group'. If a claimant is placed in the first, they are expected to take part in work focused interviews with a personal advisor. They will be given support to help them prepare for work and on gaining work will receive a work related activity component in addition to their basic rate. If the claimant is placed in the second group due to their illness or disability having a sever effect upon their ability to work, the claimant will not be expected to work at all, but can do so on a voluntary basis. These claimants will recive a support component in addition to their basic rate.

\section{Child Benefits}

(Table 10.9)

Child Benefit (CB) is paid to those responsible for children (aged under 16) or qualifying young people. The latter includes:

a) a person under the age of 19 in full-time non-advanced education or (from April 2006) on certain approved vocational training programmes

b) a person who is aged 19 who began their course of full-time, non-advanced education or approved training before reaching age 19 (note: those reaching 19 up to 9 April 2006 ceased to qualify on their 19th birthday)

c) a person who has reached the age of 16 until the 31 August following their 16th birthday

d) a person aged 16 or 17 who has left education and training who is registered with the Careers service or with Connexions and is awaiting a placement in employment or training for the limited period of up to 20 weeks from the date they left education or training. Entitlement for a qualifying young person continues until the terminal date following the date they leave full-time education or approved training. The terminal dates are at the end of August, November, February and May (there is a slight variation for Scotland). Entitlement is also maintained for a person who is entered for external examinations connected with their course throughout the period between a person leaving education or training and completing those examinations. Entitlement in all cases ceases when a person reaches the age of 20 .

Guardian's Allowance is an additional allowance for people bringing up a child because one or both of their parents has died. They must be getting Child Benefit (CB) for the child. The table shows the number of families in the UK in receipt of $C B$. The numbers shown in the table are estimates based on a random 5 per cent sample of awards current at 31 August, and are therefore subject to sampling error. The figures take no account of new claims, or revisions to claims that were received or processed after 31 August, even if they are backdated to start before 31 August.

\section{Family Credit/ Working Families' Tax Credit}

(Table 10.10)

Working Families' Tax Credit (WFTC) replaced Family Credit from 5 October 1999.

Family Credit was, and Working Families' Tax Credit is, available to families with at least one adult in remunerative work for at least 16 hours per week and who is responsible for at least one child under 16 (under 19 if in full-time education up to A-level or equivalent standard). The rate of payment of WFTC depends on the number of such children and expenditure incurred on eligible childcare. It is also higher if the worker works for at least 30 hours per week, or if there are disabled children or severely disabled adults in the family. It is tapered away above an income threshold. Further details can be obtained from HM Revenue \& Customs (HMRC).

\section{Child and Working Tax Credits (New Tax Credits)}

\section{(Table 10.11)}

Child and Working Tax Credits (CTC and WTC) replaced Working Families' Tax Credit (WFTC) from 6th April 2003. CTC and WTC are claimed by individuals, or jointly by couples, whether or not they have children.

CTC provides support to families for the children (up to the 31 August after their 16th birthday) and the 'qualifying' young people (in full-time non-advanced education until their 19th birthday) for which they are responsible. It is paid in addition to $C B$.

WTC tops up the earnings of families on low or moderate incomes. People working for at least 16 hours a week can claim it if they: (a) are responsible for at least one child or qualifying young person, (b) have a disability which puts them 
at a disadvantage in getting a job or, (c) in the first year of work, having returned to work aged at least 50 after a period of at least six months receiving out-of-work benefits. Other adults also qualify if they are aged at least 25 and work for at least 30 hours a week.

\section{Widow's Benefit and Bereavement Benefit}

\section{(Table 10.12 and 10.13)}

Widow's Benefit is payable to women widowed on or after 11 April 1988 and up to and including 8 April 2001. There are three types of Widow's Benefits: Widow's Payment, Widowed Mother's Allowance and Widow's Pension. Women widowed before 11 April 1988 continue to receive Widow's Benefit based on the rules that existed before that date. Bereavement Benefit was introduced on 9 April 2001 as a replacement for Widow's Benefit, payable to both men and women widowed on or after 9 April 2001. There are three types of Bereavement Benefits available: Bereavement Payment, Widowed Parent's Allowance and Bereavement Allowance.

\section{Government expenditure on social services and housing}

\section{(Table 10.20 to 10.25 )}

The tables of general government expenditure on social services and housing in the UK comprise a summary table followed by separate tables for each of the social services and housing categories. The definition of government expenditure used in the tables is consistent with Table 5.2.4S of the Blue Book 2009 edition, and covers both current and capital expenditure of central government (including the National Insurance Fund) and local authorities. The figures in the tables have been compiled based on the United Nations Classification of the Functions of Government (COFOG) and are consistent with the European System of Accounts 1995 (ESA95). The format of the tables was revised in the 2007 edition. As such they may not be comparable with earlier editions of the Annual Abstract of Statistics, which were based on information supplied directly by government departments. This information from government departments is generally no longer available and, as such, the tables are compiled under the categories of National Accounts.

\section{Useful links}

National Accounts Blue Book: www.statistics.gov.uk/cci/article. asp? $\mathrm{id}=2055$

UN CoFoG classification: http://unstats.un.org/unsd/cr/registry/ regcst.asp?Cl=4
The main categories of expenditure now used are:

Final Consumption Expenditure - The expenditure on goods and services that are used for the direct satisfaction of individual needs or the collective needs of members of the community as distinct from their purchase for use in the productive process. It may be contrasted with actual final consumption, which is the value of goods consumed but not necessarily purchased by that sector.

Compensation of Employees - Total remuneration payable to employees in cash or in kind. Includes the value of social contributions payable by the employer Net Procurement current expenditure less receipts for sales and charges.

Gross Capital Formation - acquisition less disposals of fixed assets and the improvement of land.

Subsidies - current unrequited payments made by general government or the European Union to enterprises. Those made on the basis of a quantity or value of goods or services are classified as 'subsidies on products'. Other subsidies based on levels of productive activity (for example, numbers employed) are designated, 'Other subsidies on production'.

Capital Transfers - transfers which are related to the acquisition or disposal of assets by the recipient or payer. They may be in cash or kind, and may be imputed to reflect the assumption or forgiveness of debt.

Non-produced financial or non financial assets - assets produced either through production or otherwise of a nonfinancial nature.

Non-market capital consumption - output of own account production of goods and services provided free or at price that are not economically significant. Non-market output is produced mainly by the general government and Non-profit Institutions Serving Household sectors.

\section{Education}

\section{(Table 10.21)}

Table 10.21 includes expenditure by the education departments, local education authorities and the University Grants Committee on education in schools, training colleges, technical institutions and universities. Compensation of employees' figures are based on revenue outturn returns produced by Department for Communities and Local Government, Welsh Assembly Government and the Scottish Government. 


\section{National Health Service}

(Table 10.22)

Table 10.22 includes expenditure by central government on hospital and community health, family practitioner and other health services. The figures are based on departmental expenditure reported to HM Treasury.

\section{Welfare services}

\section{(Table 10.23)}

Personal social services: this table covers local authority and central government expenditure on such things as the aged, handicapped, homeless, child care, care of mothers and young children, mental health, domestic help, etc.

\section{Social security}

\section{(Table 10.24)}

Table 10.24 comprises both benefits under the Social Security schemes and non-contributory benefits and allowances, administered by the Department for Work and Pensions (DWP). Benefits paid overseas are also included, as are unfunded social benefits such as voluntary employer social contributions. The analysis by type of Income Support is not exact; the estimates are derived from average numbers in receipt of benefit and average amounts paid. War pensions which are now administered by the Ministry of Defence are included in this table. Child and Working Tax Credits (NTCS) replaced Working Families' Tax Credit (WFTC) from 6 April 2003 and are administered by the HMRC.

\section{Housing}

\section{(Table 10.25)}

The table shows government expenditure on housing. It includes expenditure made by central and local government sectors, but excludes expenditure by public corporations. The Housing Revenue Account is classified as a quasipublic corporation, so that most of its current and capital expenditure and income is included in the corporate rather than government sector. All overhead and administration expenses are included in final current expenditure. Noncapitalised support for public corporations and other market bodies relating to housing is recorded as subsidies. Capital transfers are paid mainly by local government to individuals for repair and improvement of privately owned housing. Current transfers paid include insurance premiums. Gross capital formation includes that of the council houses administered by the Housing Revenue Account. This is net of any sales of housing either through Right to Buy or Large Scale Voluntary Transfers. Housing benefit in the form of rent rebates and rent allowances is not included in the table, as they are regarded as forms of social security. 
$£$ million

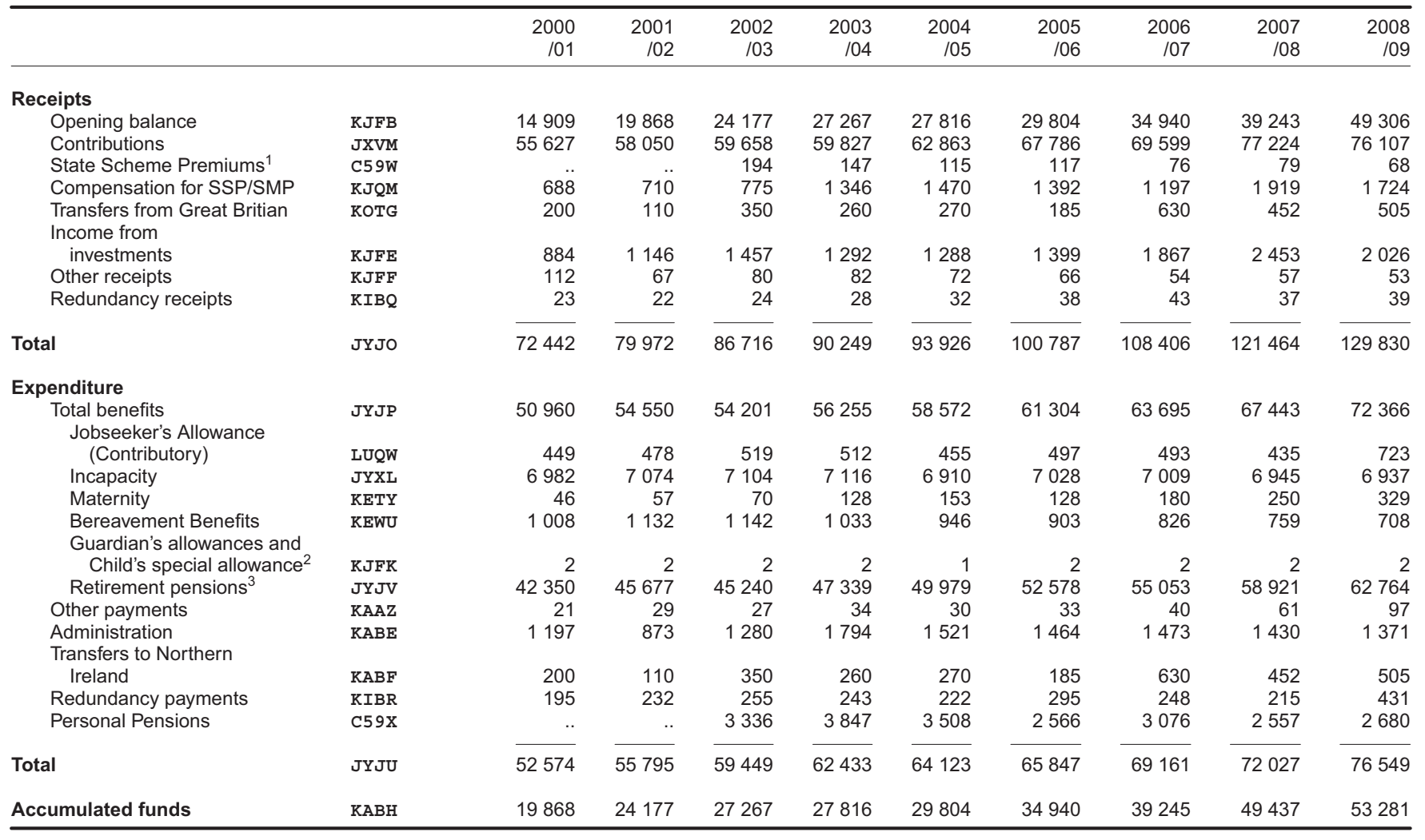

1 State Scheme Premiums are payable in respect of employed persons who cease to be covered, in certain circumstances, by a contracted out pension Sources: HM Revenue and Customs: 01702367480 scheme.

2 Includes Child's special allowance for Northern Ireland.

3 Includes personal pensions up to $2001 / 02$.

\section{Persons $^{1}$ who paid National Insurance contributions ${ }^{2,3}$ in a tax year: ${ }^{4}$ by sex United Kingdom}

\begin{tabular}{|c|c|c|c|c|c|c|c|c|c|c|c|c|}
\hline & & \multicolumn{3}{|c|}{ Total } & & \multicolumn{3}{|c|}{ Men } & & \multicolumn{3}{|c|}{ Women } \\
\hline & & $\begin{array}{r}2005 \\
106\end{array}$ & $\begin{array}{r}2006 \\
107\end{array}$ & $\begin{array}{r}2007 \\
108\end{array}$ & & $\begin{array}{r}2005 \\
106\end{array}$ & $\begin{array}{r}2006 \\
107\end{array}$ & $\begin{array}{r}2007 \\
108\end{array}$ & & $\begin{array}{r}2005 \\
106\end{array}$ & $\begin{array}{r}2006 \\
107\end{array}$ & $\begin{array}{r}2007 \\
108\end{array}$ \\
\hline Total & KABI & 29.02 & 28.91 & 28.98 & KEYF & 15.89 & 15.83 & 15.82 & KEYP & 13.13 & 13.08 & 13.17 \\
\hline Class 1 & KABJ & 24.50 & 24.50 & 24.77 & KEYG & 13.03 & 13.03 & 13.16 & KEYQ & 11.47 & 11.47 & 11.61 \\
\hline Not contracted out ${ }^{5}$ & KABK & 17.47 & 17.88 & 18.54 & KEYH & 9.69 & 9.95 & 10.35 & KEYR & 7.79 & 7.93 & 8.19 \\
\hline Contracted out & KABL & 7.03 & 6.62 & 6.23 & KEYI & 3.35 & 3.08 & 2.81 & KEYS & 3.68 & 3.54 & 3.42 \\
\hline Mixed contracted in/out ${ }^{6}$ & KABM & 1.18 & 1.10 & 1.06 & KEYJ & 0.50 & 0.48 & 0.43 & КEYT & 0.68 & 0.63 & 0.63 \\
\hline $\begin{array}{l}\text { Class } 1 \text { Reduced rate } \\
\text { (including standard rate) }\end{array}$ & $\mathrm{KABO}$ & 0.04 & 0.03 & 0.02 & KEYL & - & - & - & KEYV & 0.04 & 0.03 & 0.02 \\
\hline Class 2 exclusively ${ }^{7}$ & KABP & 2.38 & 2.40 & 2.36 & KEYM & 1.80 & 1.79 & 1.75 & KEYW & 0.59 & 0.60 & 0.62 \\
\hline Mixed Class 1 and Class 2 & KABQ & 0.71 & 0.70 & 0.70 & KEYN & 0.47 & 0.46 & 0.45 & KEYX & 0.24 & 0.25 & 0.25 \\
\hline Class 3 exclusively ${ }^{8}$ & KABR & 0.16 & 0.13 & 0.07 & KEYO & 0.07 & 0.06 & 0.03 & KEYY & 0.09 & 0.07 & 0.04 \\
\hline Mixed Class 1,2 and $3^{9}$ & I6CH & 0.06 & 0.05 & 0.01 & I6CK & 0.02 & 0.02 & - & $16 \mathrm{CN}$ & 0.03 & 0.03 & 0.01 \\
\hline
\end{tabular}

1 Based on all persons making contributions and not only if they have a quali- 5 Includes those persons with an Appropriate Personal Pension (such persons fying year. $\quad$ pay contributions at the not contracted out rate but then receive a rebate paid 2 Estimates obtained from DWP Information Directorate: Lifetime Labour directly to their scheme).

Market Data Tabulation Tool which uses a $1 \%$ sample of the National Insur- 6 Not included in the above rows.

ance Recording System (NIRS2) summer 2008 extract.

3 Components may not sum to totals as a result of rounding.

7 Persons who paid a mixture of Class 2 contributions and others are not included in this category. 4 The tax year commences on 6 April and ends on 5 April the following year. $\begin{array}{r}8 \text { Persons who paid a mixture of Class } 3 \text { contributions and others are not includ- } \\ \text { ed in this category. }\end{array}$

9 Persons with a mixture of class 1,2 or 3 contributions 


\section{Class 1}

\section{Weekly earnings \\ 2003/04 \\ Below 77.00 (LEL) \\ 77.00-89.00 (PT/ST) \\ 89.01-595.00 (UEL)}

Above 595.00 (UEL)

\section{4/05}

Below 79.00 (LEL)

79.00-91.00 (PT/ST)

91.01-610.00 (UEL)

Above 610.00(UEL)

\section{5/06}

Below 82.00 (LEL)

82.00-94.00 (PT/ST)

94.01-630.00 (UEL)

Above 630.00(UEL)

2006/07

Below 84.00 (LEL)

84.00-97.00 (PT/ST)

97.01-645.00 (UEL)

Above 645.00(UEL)

\section{7/08}

Below 87.00 (LEL)

87.00-100.00 (PT/ST)

100.01-670.00 (UEL)

Above 670.00(UEL)

2008/09

Below 90.00 (LEL)

90.00-105.00 (PT/ST)

105.01-770.00 (UEL)

Above 770.00(UEL)

\section{9/10}

Below 95.00 (LEL)

95.00-110.00 (PT/ST)

110.01-844.00 (UEL)

Above 844.00(UEL)
-
-
$11.0 \%$
$£ 55.66$

$1 \%$

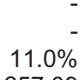

$11.0 \%$
$£ 57.09$

$\begin{array}{r}1.0 \% \\ \hline\end{array}$

-
$11.0 \%$
$£ 58.96$

$£ 58.96$

$9.4 \%$

$£ 50.38$

$1.0 \%$

See note 4

$9.4 \%$

$£ 51.51$

$11.0 \%$

$\begin{array}{r}1.0 \% \\ \hline\end{array}$

-
$11.0 \%$
$\mathbf{-} 62.70$
$1.0 \%$

$1.0 \%$
See note 4

$9.4 \%$

$£ 53.58$

$1.0 \%$
$12.8 \%$

$12.8 \%$

-

$12.8 \%$

-

$12.8 \%$

$12.8 \%$

-

$12.8 \%$

$12.8 \%$

$12.8 \%$

See note 4

$9.4 \%$

$£ 62.51$

$1.0 \%$
$9.3 \%$

$12.8 \%$

See note 6

$12.8 \%$

See note 5

$9.3 \%$

$12.8 \%$

See note 5

$9.3 \%$

$12.8 \%$

See note 5

$9.3 \%$

$12.8 \%$

\section{5}

See note 6

$9.1 \%$

$12.8 \%$

\begin{tabular}{|c|c|c|c|c|c|c|c|}
\hline \multirow[t]{2}{*}{ Above 844.00(UEL) } & \multicolumn{2}{|c|}{$1.0 \%$} & \multicolumn{2}{|c|}{$1.0 \%$} & \multicolumn{2}{|c|}{$12.8 \%$} & \multirow{2}{*}{$\begin{array}{r}12.8 \% \\
2009 / 10\end{array}$} \\
\hline & $2003 / 04$ & $2004 / 05$ & $2005 / 06$ & $2006 / 07$ & $2007 / 08$ & $2008 / 09$ & \\
\hline \multicolumn{8}{|l|}{ Class 2} \\
\hline Small earnings exception ${ }^{7}$ (per annum) & $£ 4,095$ & $£ 4,215$ & $£ 4,345$ & $£ 4,465$ & $£ 4,635$ & $£ 4,825$ & $£ 5,075$ \\
\hline \multicolumn{8}{|l|}{ Class 3} \\
\hline Flat-rate voluntary weekly contributions & $£ 6.95$ & $£ 7.15$ & $£ 7.35$ & $£ 7.55$ & $£ 7.80$ & $£ 8.10$ & $£ 12.05$ \\
\hline \multicolumn{8}{|l|}{ Class 4 (Self-employed; profit-related) } \\
\hline Rate on profits between LPL and UPL & $8.0 \%$ & $8.0 \%$ & $8.0 \%$ & $8.0 \%$ & $8.0 \%$ & $8.0 \%$ & $8.0 \%$ \\
\hline Rate on profits above UPL & $1.0 \%$ & $1.0 \%$ & $1.0 \%$ & $1.0 \%$ & $1.0 \%$ & $1.0 \%$ & $1.0 \%$ \\
\hline Lower profits limit (LPL) & $£ 4,615$ & $£ 4,745$ & $£ 4,895$ & $£ 5,035$ & $£ 5,225$ & $£ 5435$ & $£ 5715$ \\
\hline Upper profits limit (UPL) & $£ 30,940$ & $£ 31,720$ & $£ 32,760$ & $£ 33,540$ & 34,840 & $£ 40,040$ & $£ 43875$ \\
\hline
\end{tabular}

Note: LEL: Lower Earnings Limit; UEL: Upper Earnings Limit. PT: Primary Threshold; ST: Secondary Threshold.

1 Married women opting to pay contributions at the reduced rate at $3.85 \%$ before $2003-04$ and $4.85 \%$ from $2003-04$ earn no entitlement to contributory National Insurance benefits as a result of these contributions. No women have been allowed to exercise this option since 1977, but around 70,000 women who have been continually married or widowed and in the labour market since that time have retained their right to pay the reduced rate.

2 The contracted-out rebate for employees' contributions is applied only between LEL and UEL. Earnings below LEL are charged at the appropriate not contracted-out rate (which depends on total earnings). Earnings above the UEL are not subject to employee NICs before 2003-04.

3 The rates shown only apply to Contracted-Out Salary Related schemes (COSR).
Earnings below the LEL and above the UEL are charged at the appropriate not-contracted out rate. The employers' contracted-out rate applies only between the LEL and the UEL.

4 The contracted-out rebate for primary contributions is $1.6 \%$ of earnings between the LEL and the UEL for all forms of contracting-out.

5 The contracted-out rebate for secondary contributions is $3.5 \%$ of earnings between the LEL and the UEL up to 2006-07.

6 Since $2007-08$ the contracted-out rebate for secondary contributions is $3.7 \%$ of earnings between the LEL and UEL.

7 If earnings from self-employment are below this annual limit and the contributor applies for and is granted a small earnings exception Class 2 contributions need not be paid. Class 2 or 3 contributions may be paid voluntarily. 
Jobseeker's Allowance:

Personal allowances

Single

Aged under $18^{2}$

Aged $18-24$

Aged 25 or over

$\begin{array}{lllllllll}31.45 & 31.95 & 32.50 & 32.90 & 33.50 & 33.85 & 34.60 & 35.65 & 47.95\end{array}$

$\begin{array}{lllllllll}41.35 & 42.00 & 42.70 & 43.25 & 44.05 & 44.50 & 45.50 & 46.85 & 47.95\end{array}$

50.95

$\begin{array}{lllllllll}52.20 & 53.05 & 53.95 & 54.65 & 55.65 & 56.20 & 57.45 & 59.15 & 60.50\end{array}$

50.95
64.30

Lone parent

Aged under 18 - usual rate

Aged under 18 - higher rate payable in specific circumstances

$92 \mathrm{E}$

$\begin{array}{lllllllll}31.45 & 31.95 & 32.50 & 32.90 & 33.50 & 33.85 & 34.60 & 35.65 & 47.95\end{array}$

Aged 18 or over

Couple

Both aged under 18

Both under 18 , one disabled

Both under 18, with a child

One under 18, one 18 - 24

One under 18 , one $25+$

Both aged 18 or over

Dependant children and young people

Aged under 11 - 16

Aged $16-18$

Invalidity allowance

High rate

Middle rate

Low rate

Increase for dependants

Adult

Each child ${ }^{3}$

rxpt

KXDI

F92H

$\mathrm{KXDI}$

F92I

KXDM

KXD

$\mathrm{KXDP}$

KJND

KJNE

KJNF

KJNG

KJNH

Incapacity Benefit:

Short term (Lower) Under pension age

Increase for adult dependant

Short term (Lower) Over pension age

Increase for adult dependant

Short term (Higher)

Increase for dependants:

Adult

Child $^{3}$

Long term

Increase for dependants:

Adult

Child $^{3}$

Incapacity age addition: ${ }^{4}$

Higher rate

Lower rate

Employment and Support Allowance:

Single

Aged under $18^{2}$

Aged $18-24$

Aged 25 and over

Lone parent

Aged under 18 - usual rate

Aged 18 or over

KOSB

KOSC

KOSD

KOSE

KOSF

KOSG

KOSH

KOSI

Kos

KOSK

KOSL

KOSM

$\begin{array}{lllllllll}31.45 & 31.95 & 32.50 & 32.90 & 33.50 & 33.85 & 34.60 & 35.65 & 47.95\end{array}$

$\begin{array}{lllllllllll}41.35 & 42.00 & 42.70 & 43.25 & 44.05 & 44.50 & 45.50 & 46.85 & 47.95 & 50.95\end{array}$

$\begin{array}{llllllllll}62.35 & 63.35 & 64.45 & 65.30 & 66.50 & 67.15 & 68.65 & 70.70 & 72.35 & 76.90\end{array}$

$\begin{array}{llllllllll}41.35 & 42.00 & 42.70 & 43.25 & 44.05 & 44.50 & 45.50 & 46.85 & 47.95 & 50.95\end{array}$

$\begin{array}{llllllllll}52.20 & 53.05 & 53.95 & 54.65 & 55.65 & 56.20 & 57.45 & 59.15 & 60.50 & 64.30\end{array}$

$\begin{array}{llllllllll}81.95 & 83.25 & 84.65 & 85.75 & 87.30 & 88.15 & 90.10 & 92.80 & 94.95 & 100.95\end{array}$

$\begin{array}{llllllllll}26.60 & 31.45 & 33.50 & 38.50 & 42.27 & 43.88 & 45.58 & 47.45 & 52.59 & 56.11\end{array}$

$\begin{array}{llllllllll}31.75 & 32.25 & 34.30 & 38.50 & 42.27 & 43.88 & 45.58 & 47.45 & 52.59 & 56.11\end{array}$

$\begin{array}{llllllllll}14.20 & 14.65 & 14.90 & 15.15 & 15.55 & 16.05 & 16.50 & 17.10 & 17.75 & 15.65\end{array}$

$\begin{array}{llllllllll}9.00 & 9.30 & 9.50 & 9.70 & 10.00 & 10.30 & 10.60 & 11.00 & 11.40 & 9.10\end{array}$

$\begin{array}{llllllllll}4.50 & 4.65 & 4.75 & 4.85 & 5.00 & 5.15 & 5.30 & 5.50 & 5.70 & 5.35\end{array}$

$\begin{array}{llllllllll}40.40 & 41.75 & 42.45 & 43.15 & 44.35 & 45.70 & 46.95 & 48.65 & 50.55 & 53.10\end{array}$

$\begin{array}{llllllllll}11.35 & 11.35 & 11.35 & 11.35 & 11.35 & 11.35 & 11.35 & 11.35 & 11.35 & 11.35\end{array}$

$\begin{array}{llllllllll}50.90 & 52.60 & 53.50 & 54.40 & 55.90 & 57.65 & 59.20 & 61.35 & 63.75 & 67.75\end{array}$

$\begin{array}{llllllllll}31.50 & 32.55 & 33.10 & 33.65 & 34.60 & 35.65 & 36.60 & 37.90 & 39.40 & 41.35\end{array}$

$\begin{array}{llllllllll}64.75 & 66.90 & 68.05 & 69.20 & 71.15 & 73.35 & 75.35 & 78.05 & 81.10 & 86.20\end{array}$

$\begin{array}{llllllllll}38.80 & 40.10 & 42.45 & 41.50 & 42.65 & 43.95 & 45.15 & 46.80 & 48.65 & 51.10\end{array}$

$\begin{array}{llllllllll}60.20 & 62.20 & 63.25 & 64.35 & 66.15 & 68.20 & 70.05 & 72.55 & 75.40 & 80.15\end{array}$

$\begin{array}{llllllllll}31.50 & 32.55 & 33.10 & 33.65 & 34.60 & 35.65 & 36.60 & 37.90 & 39.40 & 41.35\end{array}$

$\begin{array}{llllllllll}11.35 & 11.35 & 11.35 & 11.35 & 11.35 & 11.35 & 11.35 & 11.35 & 11.35 & 11.35\end{array}$

$\begin{array}{llllllllll}67.50 & 69.75 & 70.95 & 72.15 & 74.15 & 76.45 & 78.50 & 81.35 & 84.50 & 89.80\end{array}$

$\begin{array}{llllllllll}40.40 & 41.75 & 42.45 & 43.15 & 44.35 & 45.70 & 46.95 & 48.65 & 50.55 & 53.10\end{array}$

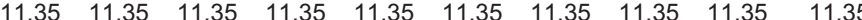

$\begin{array}{llllllllll}14.20 & 14.65 & 14.90 & 15.15 & 15.55 & 16.05 & 16.50 & 17.10 & 17.75 & 15.65\end{array}$

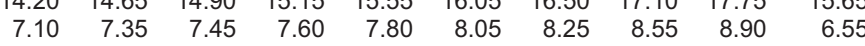

JTM6

JTM7

JTM8

JTM9

JTN2

Couple

Both aged under 18

Both under 18, with a child

Both aged under 18(main phase)

Both under 18, with a child (main phase)

One under 18 , one $18-24$

One under 18 , one $25+$

Both aged 18 or over

Attendance Allowance:

Higher rate

Lower rate

Carer's Allowance

Standard Rate

J8T6

$\begin{array}{llllll}43.15 & 44.35 & 45.70 & 46.95 & 48.65 & 50.55\end{array}$

53.10

Disability Living Allowance:

Care component

Higher rate

Middle rate

Lower rate

Mobility component

Higher rate

Lower rate
$\mathrm{KXDC}$

KXDD

$\mathrm{KXDE}$

KXDF

KXDG $\begin{array}{llllllllll}53.55 & 55.30 & 56.25 & 57.20 & 58.80 & 60.60 & 62.25 & 64.50 & 67.00 & 70.35\end{array}$ $\begin{array}{llllllllll}35.80 & 37.00 & 37.65 & 38.30 & 39.35 & 40.55 & 41.65 & 43.15 & 44.85 & 47.10\end{array}$ $\begin{array}{llllllllll}14.20 & 14.65 & 14.90 & 15.15 & 15.55 & 16.05 & 16.50 & 17.10 & 17.75 & 18.65\end{array}$

$\begin{array}{llllllllll}37.40 & 38.65 & 39.30 & 39.95 & 41.05 & 42.30 & 43.45 & 45.00 & 46.75 & 49.10\end{array}$ $\begin{array}{llllllllll}14.20 & 14.65 & 14.90 & 15.15 & 15.55 & 16.05 & 16.50 & 17.10 & 17.75 & 18.65\end{array}$ 


\begin{tabular}{|c|c|c|c|c|c|c|c|c|c|c|c|}
\hline & & 2000 & 2001 & 2002 & 2003 & 2004 & 2005 & 2006 & 2007 & 2008 & 2009 \\
\hline \multicolumn{12}{|l|}{ Maternity Benefit: } \\
\hline \multicolumn{12}{|l|}{$\begin{array}{l}\text { Maternity allowances for insured } \\
\text { women }^{6}\end{array}$} \\
\hline Lower rate ${ }^{7}$ & KJNL & 52.25 & .. & & & .. & 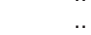 & & 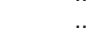 & .. & \\
\hline Standard rate & GPTJ & .. & 62.20 & 75.00 & 100.00 & 102.80 & 106.00 & 108.85 & 112.75 & 117.18 & 123.06 \\
\hline Threshold & GPTK & .. & 30.00 & 30.00 & 30.00 & 30.00 & 30.00 & 30.00 & 30.00 & 30.00 & 30.00 \\
\hline Guardian's Allowance & KJNN & 11.35 & 11.35 & 11.35 & 11.55 & 11.85 & 12.20 & 12.50 & 12.95 & 13.45 & 14.10 \\
\hline \multicolumn{12}{|l|}{ Widow's Benefit: } \\
\hline Widow's pension & KJNO & 67.50 & 72.50 & 75.50 & 77.45 & 79.60 & 82.05 & 84.25 & 87.30 & 90.70 & 95.25 \\
\hline Widowed mother's allowance & KJNP & 67.50 & 72.50 & 75.50 & 77.45 & 79.60 & 82.05 & 84.25 & 87.30 & 90.70 & 95.25 \\
\hline \multicolumn{12}{|l|}{ Bereavement Benefit: } \\
\hline Bereavement allowance & WMPF & .. & 72.50 & 75.50 & 77.45 & 79.60 & 82.05 & 84.25 & 87.30 & 90.70 & 95.25 \\
\hline Widowed parent's allowance & WMOZ & .. & 72.50 & 72.50 & 77.45 & 79.60 & 82.05 & 84.25 & 87.30 & 90.70 & 95.25 \\
\hline Addition for each child ${ }^{3}$ & WMPA & .. & 11.35 & 11.35 & 11.35 & 11.35 & 11.35 & 11.35 & 11.35 & 11.35 & 11.35 \\
\hline \multicolumn{12}{|l|}{ State Pension contributory: ${ }^{8}$} \\
\hline Single person & KJNR & 67.50 & 72.50 & 75.50 & 77.45 & 79.60 & 82.05 & 84.25 & 87.30 & 90.70 & 95.25 \\
\hline Married couple & KJNS & 107.90 & 115.90 & 120.70 & 122.80 & 127.25 & 131.20 & 134.75 & 139.60 & 145.05 & 152.30 \\
\hline \multicolumn{12}{|l|}{ State Pension non contributory: } \\
\hline Man or woman & KJNT & 40.40 & 43.40 & 45.20 & 45.45 & 47.65 & 49.15 & 50.50 & 52.30 & 54.35 & 57.05 \\
\hline Married woman & KJNU & 24.15 & 24.95 & 27.00 & 27.70 & 28.50 & 29.40 & 30.20 & 31.30 & 32.50 & 34.15 \\
\hline \multicolumn{12}{|l|}{ Industrial Injuries Benefit: } \\
\hline $\begin{array}{l}\text { Disablement pension at } 100 \text { per } \\
\text { cent rate }\end{array}$ & KJNW & 109.30 & 112.90 & 114.80 & 116.80 & 120.10 & 123.80 & 127.10 & 131.70 & 136.80 & 143.60 \\
\hline \multicolumn{12}{|l|}{ Child Benefit: } \\
\hline First child & KJOA & 15.00 & 15.50 & 15.75 & 16.05 & 16.50 & 17.00 & 17.45 & 18.10 & 18.80 & 20.00 \\
\hline \multicolumn{12}{|l|}{ War pension: } \\
\hline Ex-private (100 per cent assessment) & KJOJ & 116.00 & 116.00 & 119.80 & 121.79 & 123.90 & 127.38 & 130.20 & 133.60 & 138.34 & 152.40 \\
\hline War widow & KJOK & 87.55 & 86.74 & 89.55 & 91.00 & 92.69 & 95.27 & 98.09 & 101.43 & 105.09 & 115.55 \\
\hline
\end{tabular}




\subsection{Weekly rates of principal social security benefits ${ }^{1}$

Income Support:

Personal allowances ${ }^{9}$

Single

aged 16-17 usual rate

aged 16-17 higher rate in specific circumstances aged 18-24

\begin{tabular}{llll} 
KABS & 31.45 & 31.95 & 32.50 \\
\hline & 41.35 & 42.00 & 42.70
\end{tabular}

$\begin{array}{lll}41.35 & 42.00 & 42.70 \\ 41.35 & 42.00 & 42.70\end{array}$

32.90

$\begin{array}{lll}41.35 & 42.00 & 42.70 \\ 52.20 & 53.05 & 53.95\end{array}$

43.25
43.25

33.50

KJOY

$31.45+31.95 \quad 32.50$

54.65

Couple

both aged under 18

$\begin{array}{lll}31.45 & 31.95 & 32.50 \\ 41.35 & 42.00 & 42.70\end{array}$

KJOZ

$\begin{array}{lll}41.35 & 42.00 & 42.70 \\ 62.35 & 63.35 & 64.45\end{array}$

32.90

, one disable

$41.35 \quad 42.00 \quad 42.70$

43.25
65.30

F92 K

$\begin{array}{lll}52.20 & 53.05 & 53.95\end{array}$

F92M

One aged under 18 , one $18-24$

$\begin{array}{lll}52.20 & 53.05 & 53.95 \\ 81.95 & 83.25 & 84.65\end{array}$

43.25

One aged under 18 , one 25

KJPA

Lone parent

aged 16-17 usual rate KJPB

$\begin{array}{lll}31.45 & 31.95 & 32.50\end{array}$

54.65

85.75

44.05

44.05

33.85

44.50

45.50

55.65

56.20

45.50

35.65

46.85
46.85

47.95

$33.50 \quad 33.85$

57.45

59.15

47.95

aged 16-17 higher rate in specific circumstances

KABT

$\begin{array}{lll}41.35 & 42.00 & 42.70\end{array}$

32.90

44.05

$34.85 \quad 34.60$

35.65

$66.50 \quad 67.15$

45.50

$35.65 \quad 47.95$

44.05

$67.15 \quad 68.65$

46.85

47.95

$70.70 \quad 72.35$

$55.65 \quad 56.20$

57.45

46.85

47.95

$59.15 \quad 60.50$

88.15

90.10

92.80

64.30

aged 18 or over

$52.20 \quad 53.05 \quad 53.95$

54.65

$33.50 \quad 33.85$

$34.60 \quad 35.65 \quad 47.95$

$\begin{array}{lllll}44.05 & 44.50 & 45.50 & 46.85 & 47.95\end{array}$

57.45

$59.15 \quad 60.50$

Pension Credit $^{10}$

Standard minimum guarantee:

single

couple

Additional amount for severe disability

single

couple (one qualifies)

couple (both qualifies)

Additional amount for carers

C59Y

$\mathrm{C} 59 \mathrm{Z}$

C5A2

C5A3

C5A4

C5A8

savings credit

threshold single

threshold couple

maximum single

maximum couple

1 See chapter text

2 Persons under 18 are entitled to the appropriate adult rate.

3 The rate of child dependency increase is adjusted where it is payable for the eldest child for whom child benefit (ChB) is also paid. The weekly rate in such cases is reduced by the difference (less £3.65) between the ChB rates for the eldest and subsequent children.

4 The rate of age addition depends on age at date of onset of incapacity: higher rate for under age 35 and lower rate for age 35-44.

5 Employment and Support Allowance (ESA) replaced Incapacity Benefit and Income Support paid on the grounds of incapacity for new claims from 27 October 2008.

6 Following an EU Directive, employee's maternity benefit is aligned with the state benefit they would receive if off work sick.

7 Women who are either not employed or self-employed receive the lower rate.

8 Retirement pensioners over 80 receive $25 p$ addition.

9 In addition to personal allowances, a claimant may also be entitled to premiums. The types of premiums are family, lone parent, pensioner, higher pensioner, disability, severe disability and disabled child.

10 Pension Credit replaced Minimum Income Guarantee (MIG) for Income Support for those aged 60 and over on 6th Ocotober 2003. $\begin{array}{lllllll}102.10 & 105.45 & 109.45 & 114.05 & 119.05 & 124.05 & 130.00\end{array}$

$\begin{array}{lllllll}155.80 & 160.95 & 167.05 & 174.05 & 181.70 & 189.35 & 198.45\end{array}$

$\begin{array}{lllllll}42.95 & 44.15 & 45.50 & 46.75 & 48.45 & 50.35 & 52.85\end{array}$

$\begin{array}{lllllll}42.95 & 44.15 & 45.50 & 46.75 & 48.45 & 50.35 & 52.85\end{array}$

$\begin{array}{lllllll}85.90 & 88.30 & 91.00 & 93.50 & 96.90 & 100.70 & 105.70\end{array}$

$\begin{array}{lllllll}25.10 & 25.55 & 25.80 & 26.35 & 27.15 & 27.75 & 29.50\end{array}$

$\begin{array}{lllllll}77.45 & 79.60 & 82.05 & 84.25 & 87.30 & 91.20 & 96.00\end{array}$

$\begin{array}{rrrrrrr}123.80 & 127.25 & 131.20 & 134.75 & 139.60 & 145.80 & 153.40\end{array}$

$\begin{array}{lrrrrrr}14.79 & 15.51 & 16.44 & 17.88 & 19.05 & 19.71 & 20.40\end{array}$

\begin{tabular}{lllllll}
19.20 & 20.22 & 21.51 & 23.58 & 25.26 & 26.13 & 27.03 \\
\hline
\end{tabular}

Sources: Department for Work and Pensions Information Directorate; HM Revenue and Customs: 02074387370 Ministry of Defence/DASA (Pay \& Pensions): 02072184271 


\section{Social protection}

\section{Social Security Acts: number of persons receiving benefit ${ }^{1}$ \\ Great Britain and Overseas (excluding Northern Ireland)}

At any one time

Thousands

\begin{tabular}{|c|c|c|c|c|c|c|c|c|c|c|c|}
\hline & & 2000 & 2001 & 2002 & 2003 & 2004 & 2005 & 2006 & 2007 & 2008 & 2009 \\
\hline \multicolumn{12}{|l|}{ Persons receiving: } \\
\hline Jobseeker's Allowance ${ }^{3}$ & JYXM & 1037.01 & 909.15 & 877.38 & 885.78 & 777.40 & 800.66 & 895.88 & 807.27 & 787.87 & 1443.00 \\
\hline Employment and Support Allowance ${ }^{4}$ & JTM5 & & & & & & & & & & 288.27 \\
\hline Incapacity benefit ${ }^{2,4,5}$ & KXDT & 2352.53 & 2420.87 & 2471.15 & 2494.90 & 2508.78 & 2490.85 & 2449.99 & 2417.71 & 2382.01 & 2130.12 \\
\hline Severe Disablement Allowance & Ј8T2 & 375.56 & 374.45 & 336.48 & 320.76 & 305.94 & 292.87 & 280.01 & 267.61 & 255.56 & 244.09 \\
\hline Attendance Allowance & KXDU & 1556.10 & 1570.90 & 1290.77 & 1315.64 & 1377.35 & 1419.42 & 1465.59 & 1507.50 & 1546.68 & 1585.79 \\
\hline Disability Living Allowance & KXDW & 2193.10 & 2306.40 & 2424.35 & 2547.09 & 2644.28 & 2729.72 & 2799.16 & 2881.83 & 2973.54 & 3070.61 \\
\hline Child Benefit $^{6}$ & J8T4 & 7305.00 & 7297.10 & 7296.10 & 7297.50 & 7301.30 & 7311.40 & 7365.40 & 7449.60 & & \\
\hline Widows' Benefits & KJHF & 265.11 & 254.97 & 223.41 & 191.50 & 163.43 & 138.96 & 117.65 & 96.89 & 77.90 & 62.14 \\
\hline Bereavement Benefits & VQAA & .. & .. & 41.49 & 47.68 & 51.18 & 55.24 & 57.66 & 58.54 & 59.85 & 61.91 \\
\hline \multicolumn{12}{|l|}{ National Insurance } \\
\hline \multicolumn{12}{|l|}{ State pension contributory: } \\
\hline Males $^{2}$ & $\mathrm{KJHH}$ & 4039.40 & 4083.90 & 4149.15 & 4211.36 & 4275.68 & 4336.81 & 4374.17 & 4432.29 & 4520.56 & 4626.96 \\
\hline Females $^{2}$ & KJHL & 6928.00 & 6959.70 & 6972.19 & 7037.15 & 7117.78 & 7197.93 & 7245.69 & 7391.11 & 7529.40 & 7650.40 \\
\hline Total $^{2}$ & KJHG & 10967.40 & 11043.60 & 11121.34 & 11248.52 & 11393.45 & 11534.73 & 11619.88 & 11823.40 & 12049.97 & 12277.36 \\
\hline Males & KJHI & 5.20 & 5.10 & 5.26 & 5.37 & 5.39 & 5.34 & 5.36 & 5.68 & 6.23 & 6.67 \\
\hline Females & KJHJ & 18.00 & 18.20 & 18.06 & 17.73 & 17.31 & 16.74 & 16.58 & 17.34 & 18.80 & 20.04 \\
\hline Total & KJHK & 23.20 & 23.30 & 23.32 & 23.10 & 22.70 & 22.08 & 21.94 & 23.03 & 25.03 & 26.71 \\
\hline \multicolumn{12}{|l|}{ Industrial Injuries Disablement ${ }^{2,7}$} \\
\hline Pensions assessments ${ }^{5}$ & KJHN & 274.60 & 275.40 & 273.70 & 267.13 & 266.48 & 267.12 & 266.45 & 264.88 & 262.73 & 260.69 \\
\hline \multicolumn{12}{|l|}{ Reduced Earnings Allowance/ } \\
\hline Retirement Allowance assessments ${ }^{8}$ & KEYC & 82.90 & 82.60 & 81.00 & 76.22 & 74.81 & 73.15 & 71.38 & 69.36 & 67.19 & 65.16 \\
\hline Income Support (Excluding MIG) & KABV & 2237.13 & 2260.63 & 2238.76 & 2236.38 & 2192.64 & 2139.78 & 2114.77 & 2117.70 & 2091.52 & 1979.80 \\
\hline Minimum Income Guaranteed & J8T5 & 1607.48 & 1714.37 & 1737.53 & 1777.79 & 12.09 & 10.98 & 10.27 & 10.65 & 10.74 & 10.19 \\
\hline Pension Credit & C5AP & .. & .. & .. & .. & 2490.76 & 2682.73 & 2717.39 & 2733.50 & 2719.14 & 2730.56 \\
\hline \multicolumn{12}{|l|}{ Housing Benefit and Council Tax Benefit } \\
\hline Housing Benefit Total ${ }^{9,10}$ & EW3X & 4033.30 & 3874.40 & 3812.63 & 3796.42 & 3879.42 & 3956.82 & 3990.03 & 4031.81 & .. & 4412.99 \\
\hline Social Landlord ${ }^{11}$ & KABY & 3218.35 & 3131.14 & 3093.80 & 3081.67 & 3135.49 & 3165.89 & 3152.25 & 3108.73 & .. & 3186.40 \\
\hline Private Landlord & KABZ & 814.95 & 743.26 & 718.83 & 714.75 & 743.93 & 790.93 & 837.79 & 923.07 & .. & 1221.42 \\
\hline Council tax benefit ${ }^{12}$ & KJPO & 4830.06 & 4673.37 & 4601.73 & 4627.78 & 4800.22 & 4959.69 & 5049.97 & 5076.94 & & 5440.06 \\
\hline War pensions $^{13}$ & KADG & 295.67 & 284.33 & 272.78 & 260.79 & 247.59 & 235.30 & 223.85 & 212.54 & 201.27 & 190.75 \\
\hline
\end{tabular}

1 See chapter text. Figures as at May each year unless otherwise stated.

2 Due to rounding errors several figures have been revised for May 2008.
3 Totals include 'credits only' cases.

Sources: DWP Information Directorate: Work and Pensions Longitudinal Study

4 Employment and Support Allowance (ESA) replaced Incapacity Benefit and

Income Support paid on the grounds of incapacity for new claims from 27th

HM Revenue and Customs: 02074387370 ;

October 2008

5 Totals also include 'Credits only' cases.

6 Figures for Child Benefit in 2008 and 2009 are delayed due to extraction

system updates.

7 Figures for IIDB include those receiving both IIDB and REA, at March.

8 Figures show REA cases only and are at March each year.

9 The DWP have implemented an improvement to the way in which HB and

CTB caseload statistics are compiled. Historic statistics for the period up to August 2008 are based on clerical returns made by Local Authorities (LAs) of the aggregate number of people claiming HB and CTB as a specific point in time. This has gradually transitioned into a monthly electronic scan of claimant level data direct from the LA computer systems. This data source (the Single Housing Benefit Extract (SHBE)) has been designed to provide sufficient information for all current and future statistical purposes and is now the single source of $\mathrm{HB}$ and CTB data.

10 Housing Benefit figures excludes any Extended Payment cases.

11 Social landlord figures include registered social landlord tenants.

12 Figure excludes Second Adult Rebate Claims.

13 Figures for War pensions are at March each year. 
Social protection

Jobseeker's Allowance ${ }^{1,2,3}$ claimants: by benefit entitlement

Great Britain and Overseas (excluding Northern Ireland)

As at May

2003

2004

2005

2006

Thousands

All Persons

All with benefit - total

Contribution-based JSA only

Contribution based JSA \& income-based JSA

Income-based JSA only payment

No benefit in payment

Total

$\begin{array}{lrrrrrrr}\text { KXDX } & 797.9 & 699.6 & 728.3 & 812.0 & 730.8 & 718.0 & 1316.4 \\ \text { KXDY } & 160.4 & 131.0 & 139.5 & 134.6 & 113.6 & 127.8 & 341.8 \\ \text { KXDZ } & 18.1 & 13.5 & 13.5 & 13.0 & 11.9 & 12.8 & 34.6 \\ \text { KXEA } & 619.4 & 555.1 & 575.3 & 664.5 & 605.3 & 577.4 & 940.0 \\ \text { KXEB } & 87.9 & 77.8 & 72.4 & 83.9 & 76.4 & 69.9 & 126.6 \\ & & & & & & & \\ \text { KXEC } & 885.8 & 777.4 & 800.7 & 895.9 & 807.4 & 788.0 & 1443.0\end{array}$

\section{Males}

All with benefit - total

Contribution-based JSA only

Contribution based JSA \& income-based JSA

Income-based JSA only payment

No benefit in payment

KXED

KXEE
KXEF

605.6

KXEF

114.1
15.9

KXEG

475.6

KXEH

60.3

527.2
93.8

93.8
12.3

421.1

52.7

KXEI

665.9

580.0

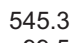

606.8

537.8

529.9

$529.9 \quad 978.9$

12.6

433.2

12.0

79.6
10.7

11.7

248.7
31.2

$\begin{array}{llll}498.9 & 447.5 & 427.6 & 698.9\end{array}$

Total

595.1

663.4

589.6

576.7

88.8

Females

All with benefit - total

Contribution-based JSA only

Contribution based JSA \& income-based JSA

Income-based JSA only payment

No benefit in payment

KXEJ 192

KXEK $\quad 192.3$

KXEK

46.3
2.2

KXEM

2.2
143.8

172.4

182.9205

$37.2 \quad 40.0$

1.2

134.0

0.8
142.1

205.3
38.7

1.0
165.5

193.0

KXEN

219.8

197.4

205.5

232.5

1.2
157.8

157.8
24.8

188.1

37.2

1.2
149.8

23.2

337.6

93.1

241.1

KXEO

1 See chapter text. Jobseeker's Allowance (JSA) has two routes of entry: contribution-based which depends mainly upon national insurance contribu-
tions and income-based which depends mainly on a means test. Some Figures are given at May each year and have been derived by applying $5 \%$ proportions to $100 \%$ totals taken from the DWP $100 \%$ Work and Pensions Longitudinal Study (WPLS).

claimants can qualify by either route. In practice they receive income-based 3 Figures are rounded to the nearest hundred and quoted in thousands. They JSA but have an underlying entitlement to the contribution-based element.

not sum due to rounding. 


\begin{tabular}{|c|c|c|c|c|c|c|c|}
\hline & & 2004 & 2005 & 2006 & 2007 & $2008^{4}$ & 2009 \\
\hline \multicolumn{8}{|l|}{ Males } \\
\hline All durations: All ages & KJJA & 1517.62 & 1492.38 & 1455.52 & 1428.65 & 1399.58 & 1419.43 \\
\hline Under 20 & KJJB & 22.04 & 21.45 & 19.95 & 18.66 & 17.25 & 18.09 \\
\hline $20-29$ & KJJC & 142.68 & 143.24 & 141.80 & 146.07 & 149.47 & 159.10 \\
\hline $30-39$ & KJJD & 253.32 & 245.61 & 233.70 & 224.29 & 215.51 & 215.95 \\
\hline $40-49$ & KJJE & 318.04 & 320.77 & 319.77 & 320.24 & 319.22 & 330.96 \\
\hline $50-59$ & KJJF & 463.37 & 451.93 & 439.54 & 418.26 & 404.76 & 405.59 \\
\hline $60-64$ & KJJG & 318.12 & 309.36 & 300.73 & 301.10 & 293.33 & 289.57 \\
\hline 65 and over & KJJH & 0.05 & 0.04 & 0.02 & 0.03 & 0.04 & 0.17 \\
\hline Over six months: All ages & KJJI & 1359.08 & 1347.43 & 1323.20 & 1291.32 & 1266.80 & 1253.91 \\
\hline Under 20 & KJJJ & 13.78 & 13.51 & 12.85 & 11.70 & 10.90 & 10.42 \\
\hline $20-29$ & KJJK & 110.85 & 114.57 & 115.21 & 117.83 & 121.90 & 124.50 \\
\hline $30-39$ & KJJL & 217.81 & 213.91 & 205.36 & 195.22 & 188.25 & 182.41 \\
\hline $40-49$ & KJJM & 285.90 & 290.72 & 291.36 & 289.94 & 289.72 & 293.13 \\
\hline $50-59$ & KJJN & 427.06 & 418.60 & 409.46 & 387.76 & 374.75 & 368.48 \\
\hline $60-64$ & KJJO & 303.64 & 296.10 & 288.93 & 288.85 & 281.25 & 274.93 \\
\hline 65 and over & KJJP & 0.04 & 0.02 & 0.02 & 0.03 & 0.03 & 0.05 \\
\hline \multicolumn{8}{|l|}{ Females } \\
\hline All durations: All ages & KJJQ & 990.84 & 998.20 & 994.33 & 988.93 & 982.33 & 998.74 \\
\hline Under 20 & KJJR & 21.48 & 20.51 & 18.92 & 17.86 & 16.79 & 15.68 \\
\hline $20-29$ & KJJS & 105.02 & 108.61 & 109.73 & 114.42 & 117.91 & 121.50 \\
\hline $30-39$ & КJJT & 177.91 & 173.45 & 167.36 & 162.39 & 156.95 & 156.85 \\
\hline $40-49$ & KJJU & 270.90 & 276.62 & 279.32 & 283.45 & 285.84 & 296.87 \\
\hline $50-59$ & KJJV & 415.52 & 418.99 & 418.99 & 410.80 & 404.82 & 407.82 \\
\hline 60 and over & KJJW & 0.02 & 0.02 & 0.02 & 0.02 & 0.02 & 0.02 \\
\hline Over six months: All ages & KJJX & 880.52 & 894.57 & 896.33 & 885.69 & 881.41 & 882.94 \\
\hline Under 20 & KJJY & 12.40 & 12.10 & 11.13 & 10.20 & 9.55 & 8.75 \\
\hline $20-29$ & KJJZ & 84.02 & 88.98 & 90.99 & 93.60 & 97.24 & 99.15 \\
\hline $30-39$ & KJKA & 154.95 & 152.48 & 148.00 & 142.28 & 137.59 & 134.90 \\
\hline $40-49$ & KJKB & 243.52 & 250.11 & 253.50 & 255.99 & 258.74 & 263.70 \\
\hline $50-59$ & KJKC & 385.61 & 390.88 & 392.69 & 383.60 & 378.27 & 376.43 \\
\hline 60 and over & KJKD & 0.02 & 0.02 & 0.02 & 0.02 & 0.02 & 0.02 \\
\hline \multicolumn{8}{|l|}{ Unknown Gender } \\
\hline All durations & EW4 4 & 0.31 & 0.26 & 0.15 & 0.13 & 0.11 & 0.23 \\
\hline Over 6 months & EW4 5 & 0.16 & 0.13 & 0.10 & 0.09 & 0.09 & 0.10 \\
\hline
\end{tabular}

Definitions and conventions. Caseload figures are rounded to the near- 3 From 27th October 2008, new claims to Incapacity Benefit can also be allocatest ten and displayed in thousands. Totals may not sum due to rounding. ed, on incapacity grounds, to the newly introduced Employment and Support 1 See chapter text. Figures are given at May each year. Allowance (ESA).

2 Table includes Employment and Support Allowance and Incapacity Benefit 4 Due to rounding errors several figures have been revised for May 2008.

ONLY claimants and not those claiming Severe Disablement Allowance (SDA).

Sources: Department for Work and Pensions, Information Directorate

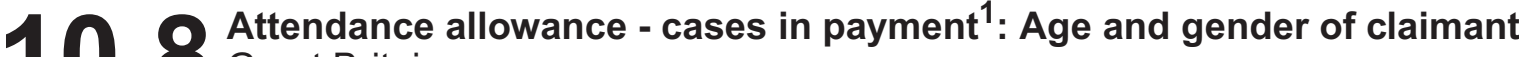 Great Britain At May each year}

Thousands

\begin{tabular}{|c|c|c|c|c|c|c|c|c|}
\hline & & 2003 & 2004 & 2005 & 2006 & 2007 & 2008 & 2009 \\
\hline Males: All ages & JT9Z & 393.9 & 418.5 & 436.9 & 459.5 & 478.4 & 497.2 & 516.5 \\
\hline Unknown age & JTA2 & - & - & - & - & - & - & - \\
\hline $65-69$ & JTA3 & 19.5 & 21.4 & 22.0 & 22.3 & 22.8 & 23.5 & 24.4 \\
\hline $70-74$ & JTA4 & 56.6 & 59.8 & 61.6 & 64.2 & 66.8 & 70.2 & 73.7 \\
\hline $75-79$ & JTA5 & 100.9 & 103.8 & 104.2 & 104.8 & 106.3 & 109.1 & 112.4 \\
\hline $80-84$ & JTA6 & 110.1 & 121.7 & 125.3 & 130.4 & 133.1 & 135.4 & 137.8 \\
\hline $85-89$ & JTA7 & 68.5 & 70.1 & 78.7 & 89.4 & 98.5 & 107.7 & 116.0 \\
\hline Females: All ages & JTA9 & 921.8 & 958.9 & 982.6 & 1006.2 & 1029.1 & 1049.5 & 1069.3 \\
\hline Unknown age & JTB2 & 0.1 & - & - & - & - & - & - \\
\hline $65-69$ & JTB3 & 25.1 & 27.3 & 27.7 & 28.3 & 28.4 & 29.1 & 30.0 \\
\hline $70-74$ & JTB4 & 88.2 & 91.5 & 92.0 & 93.6 & 96.4 & 99.6 & 103.5 \\
\hline $75-79$ & JTB5 & 189.1 & 190.9 & 189.1 & 186.8 & 185.8 & 186.7 & 188.4 \\
\hline $80-84$ & JTB6 & 260.7 & 282.5 & 282.0 & 279.4 & 278.3 & 277.7 & 277.7 \\
\hline $85-89$ & JTB7 & 206.1 & 204.7 & 221.4 & 241.6 & 259.3 & 276.7 & 290.9 \\
\hline
\end{tabular}

1 Totals show the number of people in receipt of allowance, and exclude peo-

ple with entitlement where the payment has been suspended, for example if they are in hospital. 


\begin{tabular}{|c|c|c|c|c|c|c|c|c|c|c|c|}
\hline & & \multicolumn{10}{|c|}{$\begin{array}{l}\text { United Kingdom } \\
\text { As at } 31 \text { August }\end{array}$} \\
\hline & & 2000 & 2001 & 2002 & 2003 & 2004 & 2005 & 2006 & 2007 & 2008 & 2009 \\
\hline \multicolumn{12}{|l|}{ Families receiving allowances: ${ }^{2}$} \\
\hline Total & vowx & 7340 & 7335 & 7336 & 7246 & 7296 & 7315 & 7413 & 7475 & 7583 & 7770 \\
\hline With 1 child & vowY & 3128 & 3143 & 3162 & 3067 & 3165 & 3187 & 3266 & 3345 & 3468 & 3606 \\
\hline 2 children & vowz & 2898 & 2891 & 2894 & 2907 & 2891 & 2891 & 2910 & 2904 & 2903 & 2936 \\
\hline 4 or more children & voxB & 251 & 247 & 242 & 325 & 315 & 316 & 318 & 317 & 313 & 320 \\
\hline Families receiving Guardian's Allowance ${ }^{3}$ & voxH & 2.5 & 2.3 & 2.5 & 2.6 & 2.9 & 2.8 & 3.2 & 3.3 & .. & .. \\
\hline
\end{tabular}

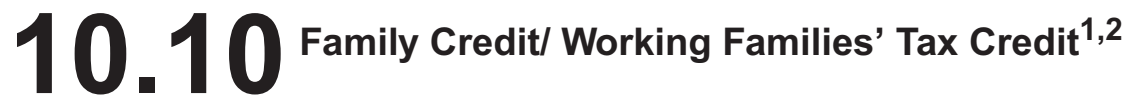

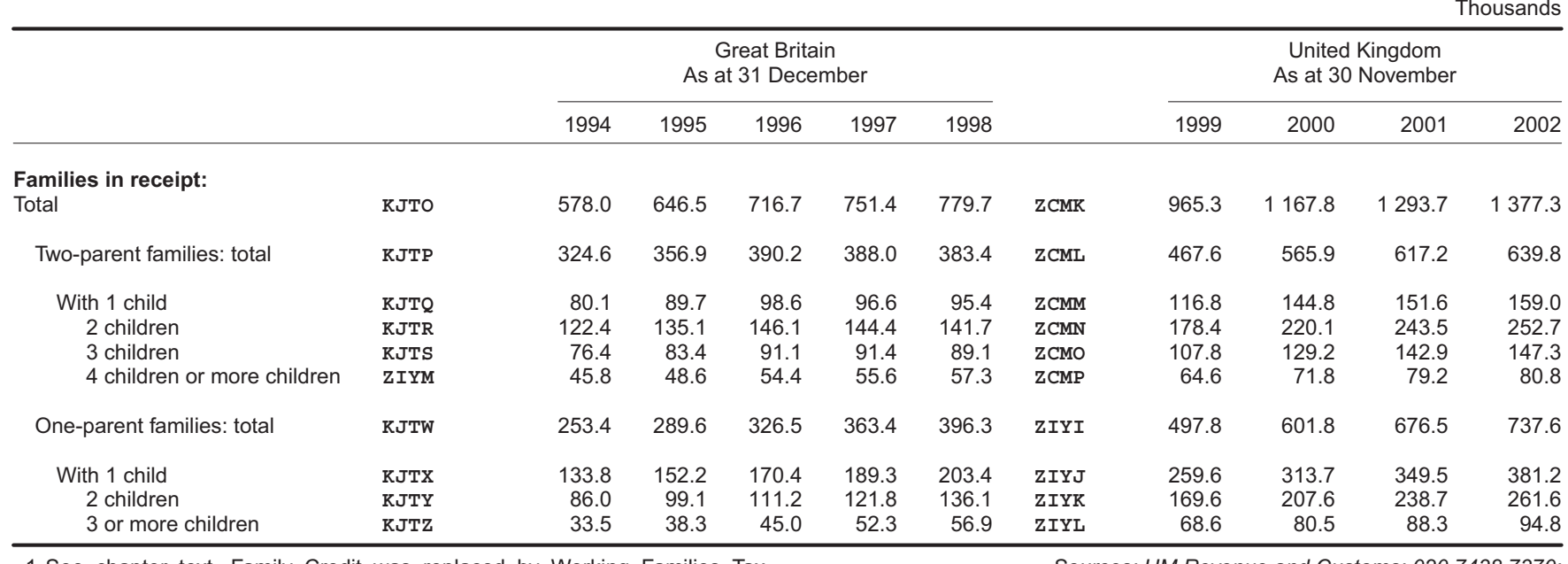

1 See chapter text. Family Credit was replaced by Working Families Tax Credit (WFTC) in October 1999. The WFTC figures for December 1999 include Family Credit awards made before October 1999 and still current Sources: HM Revenue and Customs: 0207438 7370; Department for Work and Pensions; (both FC and WFTC awards last for 26 weeks).

2 WFTC was replaced by Child Credit and Working Tax Credit on 6th April 2003. See table 10.11.

\subsection{In-work families with Child Tax Credit or Working Tax Credit awards \\ As at December}

Thousands

\begin{tabular}{|c|c|c|c|c|c|c|c|c|}
\hline & & $2003^{1}$ & 2004 & 2005 & 2006 & 2007 & 2008 & 2009 \\
\hline In-work families with positive award: & C5PF & 4423 & 4519 & 4538 & 4526 & 4541 & 4630 & 4712 \\
\hline With children & C5PG & 4208.0 & 4261.0 & 4218.0 & 4204.0 & 4189.0 & 4205.0 & 4200.0 \\
\hline Receiving Working Tax Credit and Child Tax Credit & $\mathrm{C} 5 \mathrm{PH}$ & 1548.0 & 1492.0 & 1497.0 & 1596.0 & 1650.0 & 1763.0 & 1870.0 \\
\hline Receiving Child Tax Credit only & C5PI & 2660.0 & 2769.0 & 2721.0 & 2608.0 & 2539.0 & 2442.0 & 2330.0 \\
\hline \multicolumn{9}{|l|}{ Without children } \\
\hline Working Tax Credit only & C5PL & 215.0 & 258.0 & 320.0 & 323.0 & 352.0 & 426.0 & 511.0 \\
\hline
\end{tabular}

1 Child and Working Tax Credits replaced Working Families' Tax Credit on 6th April 2003. Figures for 2003 are based on awards current at 5th Jan-

uary 2004. All other figures at December each year. See chapter text. 


\begin{tabular}{|c|c|c|c|c|c|c|c|}
\hline & & 2004 & 2005 & 2006 & 2007 & 2008 & 2009 \\
\hline \multicolumn{8}{|c|}{$\begin{array}{l}\text { All Widows' Benefit (excluding } \\
\text { bereavement allowance) }\end{array}$} \\
\hline All ages & KJGA & 163.40 & 139.00 & 117.70 & 96.89 & 77.90 & 62.14 \\
\hline Unknown Age & EW4O & - & - & - & 0.02 & - & - \\
\hline $18-24$ & EW4P & - & - & - & - & - & - \\
\hline $25-29$ & EW4Q & 0.20 & 0.10 & 0.10 & 0.04 & 0.02 & 0.01 \\
\hline $30-34$ & EW4R & 1.20 & 0.80 & 0.50 & 0.32 & 0.20 & 0.13 \\
\hline $40-44$ & EW4T & 7.50 & 6.10 & 4.90 & 3.93 & 3.04 & 2.31 \\
\hline $45-49$ & EW4U & 13.20 & 11.00 & 9.10 & 7.58 & 6.26 & 5.14 \\
\hline $50-54$ & EW4V & 33.30 & 26.90 & 21.80 & 17.69 & 14.42 & 11.72 \\
\hline $55-59$ & EW4W & 77.70 & 66.90 & 57.30 & 45.78 & 36.86 & 30.37 \\
\hline $60-64$ & EW4X & 26.40 & 24.30 & 21.80 & 20.01 & 16.01 & 11.71 \\
\hline \multicolumn{8}{|c|}{$\begin{array}{l}\text { Widowed parents' allowance - } \\
\text { with dependant children }\end{array}$} \\
\hline All ages & KJGG & 28.20 & 23.20 & 19.00 & 15.60 & 12.60 & 9.98 \\
\hline $18-24$ & EW4 Z & - & - & - & - & - & - \\
\hline $25-29$ & EW52 & 0.20 & 0.10 & 0.10 & 0.03 & 0.02 & 0.01 \\
\hline $30-34$ & EW53 & 1.10 & 0.80 & 0.50 & 0.31 & 0.19 & 0.12 \\
\hline $35-39$ & EW54 & 3.80 & 2.80 & 2.10 & 1.49 & 1.05 & 0.72 \\
\hline $40-44$ & EW55 & 7.00 & 5.70 & 4.60 & 3.75 & 2.92 & 2.23 \\
\hline $45-49$ & EW56 & 7.80 & 6.70 & 5.60 & 4.71 & 3.87 & 3.13 \\
\hline $50-54$ & EW57 & 5.70 & 4.80 & 4.10 & 3.58 & 3.10 & 2.53 \\
\hline $55-59$ & EW58 & 2.30 & 2.00 & 1.80 & 1.57 & 1.33 & 1.13 \\
\hline $60-64$ & EW59 & 0.30 & 0.30 & 0.20 & 0.17 & 0.13 & 0.10 \\
\hline \multicolumn{8}{|c|}{$\begin{array}{l}\text { Widowed parents' allowance - } \\
\text { without dependant children }\end{array}$} \\
\hline All ages & KJGM & 1.40 & 1.10 & 0.80 & 0.69 & 0.54 & 0.46 \\
\hline Unknown Age & EW5A & - & - & - & - & - & - \\
\hline $18-24$ & EW5B & - & - & - & - & - & - \\
\hline $25-29$ & EW5C & - & - & - & - & - & - \\
\hline $30-34$ & EW5D & - & - & - & 0.01 & 0.01 & 0.01 \\
\hline $35-39$ & EW5E & 0.10 & 0.10 & 0.10 & 0.04 & 0.03 & 0.02 \\
\hline $50-54$ & EW5H & 0.30 & 0.30 & 0.20 & 0.17 & 0.13 & 0.13 \\
\hline $55-59$ & EW5I & 0.20 & 0.20 & 0.10 & 0.11 & 0.10 & 0.07 \\
\hline $60-64$ & EW5J & - & - & - & 0.02 & 0.01 & 0.01 \\
\hline \multicolumn{8}{|c|}{ Age -related bereavement allowance } \\
\hline All ages & KJGS & 110.10 & 96.60 & 84.00 & 70.13 & 57.37 & 46.58 \\
\hline Unknown Age & EW5K & - & - & - & 0.01 & - & - \\
\hline $18-24$ & EW5L & - & - & - & - & - & - \\
\hline $25-29$ & EW5M & - & - & - & - & - & - \\
\hline $30-34$ & EW5N & - & - & - & - & - & - \\
\hline $35-39$ & EW50 & - & - & - & - & - & - \\
\hline $40-44$ & EW5P & 0.20 & 0.20 & 0.10 & 0.06 & 0.03 & 0.01 \\
\hline $45-49$ & EW5Q & 5.10 & 4.00 & 3.30 & 2.66 & 2.23 & 1.86 \\
\hline $50-54$ & EW5R & 26.70 & 21.40 & 17.20 & 13.75 & 11.08 & 9.01 \\
\hline $55-59$ & EW5S & 66.30 & 59.00 & 50.90 & 40.57 & 32.61 & 26.83 \\
\hline $60-64$ & EW5T & 11.90 & 12.00 & 12.50 & 13.08 & 11.42 & 8.87 \\
\hline \multicolumn{8}{|c|}{ Bereavement allowance (Not age related) } \\
\hline All ages & KJGW & 23.70 & 18.10 & 13.90 & 10.47 & 7.39 & 5.12 \\
\hline Unknown Age & EW5U & - & - & - & - & - & - \\
\hline $18-24$ & EW5V & - & - & - & - & - & - \\
\hline $25-29$ & EW5W & - & - & - & - & - & - \\
\hline $30-34$ & EW5X & - & - & - & - & - & - \\
\hline $35-39$ & EW5Y & - & - & - & - & - & - \\
\hline $40-44$ & EW5Z & - & - & - & - & - & - \\
\hline $45-49$ & EW62 & - & - & - & - & - & - \\
\hline $50-54$ & EW63 & 0.60 & 0.50 & 0.30 & 0.20 & 0.11 & 0.05 \\
\hline $55-59$ & EW64 & 8.80 & 5.60 & 4.40 & 3.53 & 2.82 & 2.33 \\
\hline $60-64$ & EW65 & 14.20 & 12.00 & 9.10 & 6.74 & 4.45 & 2.74 \\
\hline
\end{tabular}

1 Definitions and Conventions: "-" Nil or Negligible; "." Not applicable; 3 Figures include overseas cases.

Caseload figures are rounded to the nearest hundred and displayed in thousands.

2 Caseload (Thousands) All Claimants of Widows Benefit are female. No new claims for WB have been accepted since April 2001 when it was replaced

Sources: DWP Information Directorate: Work and Pensions Longitudinal Study $100 \%$ data; by Bereavement Benefit. 
Social protection

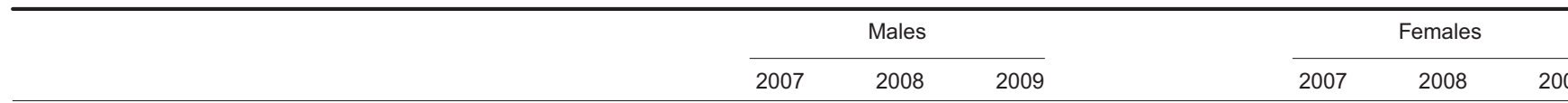

All Bereavement Benefit (excluding bereavement allowance)

All ages

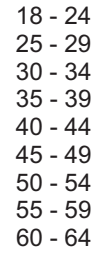

Widowed parents' allowance with dependant children All ages

$$
\begin{aligned}
& 18-24 \\
& 25-29 \\
& 30-34 \\
& 35-39 \\
& 40-44 \\
& 45-49 \\
& 50-54 \\
& 55-59 \\
& 60-64
\end{aligned}
$$

Widowed parents' allowance without dependant children All ages

$$
\begin{aligned}
& 18-24 \\
& 25-29 \\
& 30-34 \\
& 35-39 \\
& 40-44 \\
& 45-49 \\
& 50-54 \\
& 55-59 \\
& 60-64
\end{aligned}
$$

Age-related bereavement allowance All ages

$$
\begin{aligned}
& 18-24 \\
& 25-29 \\
& 30-34 \\
& 35-39 \\
& 40-44 \\
& 45-49 \\
& 50-54 \\
& 55-59 \\
& 60-64
\end{aligned}
$$

Bereavement allowance (not age related) All ages

$$
\begin{aligned}
& 18-24 \\
& 25-29 \\
& 30-34 \\
& 35-39 \\
& 40-44 \\
& 45-49 \\
& 50-54 \\
& 55-59
\end{aligned}
$$

\begin{tabular}{|c|c|c|c|c|c|c|c|}
\hline WLSX & 17.77 & 17.82 & 18.60 & WLTC & 40.77 & 42.04 & 43.32 \\
\hline EVW9 & & & & EVY2 & 0.07 & 0.08 & 0.06 \\
\hline EVX2 & 0.05 & 0.06 & 0.06 & EVY3 & 0.53 & 0.53 & 0.53 \\
\hline EVX3 & 0.28 & 0.25 & 0.25 & EVY4 & 1.63 & 1.66 & 1.68 \\
\hline EVX4 & 1.15 & 1.11 & 1.06 & EVY5 & 4.05 & 4.31 & 4.30 \\
\hline EVX5 & 2.50 & 2.50 & 2.47 & EVY6 & 7.01 & 7.33 & 7.61 \\
\hline EVX6 & 3.61 & 3.69 & 3.92 & EVY7 & 8.92 & 9.54 & 10.22 \\
\hline EVX7 & 3.47 & 3.51 & 3.68 & EVY8 & 8.65 & 9.10 & 9.64 \\
\hline EVX8 & 3.43 & 3.33 & 3.38 & EVY9 & 9.90 & 9.49 & 9.28 \\
\hline EVX9 & 3.29 & 3.36 & 3.77 & EVZ2 & . & & \\
\hline
\end{tabular}

\begin{tabular}{|c|c|c|c|c|c|c|c|}
\hline WLUD & 11.27 & 11.51 & 11.81 & WLUH & 26.86 & 29.18 & 31.08 \\
\hline EVZ3 & & & & EW2 4 & 0.07 & 0.08 & 0.06 \\
\hline EVZ4 & 0.05 & 0.06 & 0.06 & EW2 5 & 0.52 & 0.52 & 0.53 \\
\hline EVZ5 & 0.28 & 0.25 & 0.25 & EW2 6 & 1.61 & 1.64 & 1.66 \\
\hline EVZ6 & 1.14 & 1.11 & 1.06 & EW2 7 & 4.01 & 4.27 & 4.26 \\
\hline EVZ7 & 2.48 & 2.49 & 2.46 & EW2 8 & 6.93 & 7.25 & 7.54 \\
\hline EVZ8 & 3.13 & 3.26 & 3.39 & EW29 & 7.23 & 7.99 & 8.70 \\
\hline EVZ9 & 2.36 & 2.48 & 2.62 & EW2A & 4.61 & 5.29 & 5.97 \\
\hline EW22 & 1.29 & 1.32 & 1.38 & EW2B & 1.89 & 2.14 & 2.36 \\
\hline EW23 & 0.53 & 0.54 & 0.59 & EW2C & .. & .. & . \\
\hline
\end{tabular}$$
60-64
$$

1 Figures include overseas cases.

2 Figures are given at May each year and are taken from the DWP $100 \%$ Work and Pensions Longitudinal Study (WPLS).
WLVK

EW2D

EW2E

EW2F

EW2 $\mathrm{H}$

EW2 I

EW2 J

EW2K

EW2L

WMOB

EW2V

EW2W

EW2X

EW2Y

EW2 Z

EW3 2

EW3 3

EW3 4

EW3 5

WMOX

EW3 F

EW3G

EW3H

EW3 I

EW3 J

EW3K

EW3 I

EW3M

EW3N
0.05

0.04

WMMR

EW2M

EW2N

EW2O

EW2P

EW2Q

EW2 R

EW2S

EW2 T

EW2U

$1.71 \quad 1.59$

WMOC

EW3 6

EW3 7

EW3 8

EW39

EW3A

EW3B

EW3C

EW3D

EW3E

$\begin{array}{rrrr}1.10 & 1.03 & 1.06 & \text { EW3C } \\ 0.15 & 0.14 & 0.16 & \text { EW3D } \\ . . & . . & . . & \text { EW3E }\end{array}$

$\begin{array}{lll}4.74 & 4.68 & 5.01\end{array}$

EW30

EW3P

EW3Q

EW3R

EW3S

EW3 T

EW3U

EW3V

1.86
2.81

3.18

\begin{tabular}{lll}
1.98 & 2.86 & 3.18 \\
\hline
\end{tabular}

EW3W

Sources: Department for Work and Pensions; Work and Pensions Longitudinal Study (WPLS); Information Directorate 


\section{Contributory and non-contributory retirement pensions: $:^{1,2}$ by sex and \\ age of claimant}

Great Britain and Overseas. At May each year.

Thousands and percentages

2005

2006

2008

2009

Men:

$\begin{array}{ll}\text { Age-groups: } & \\ \text { 65-69 } & \text { KJSB } \\ \text { Percentage } & \text { KJSC } \\ \text { 70-74 } & \text { KJSD } \\ \text { Percentage } & \text { KJSE } \\ 75-79 & \text { KJSF } \\ \text { Percentage } & \text { KJSG } \\ \text { 80-84 } & \text { KJSH } \\ \text { Percentage } & \text { KJSI } \\ \text { 85-89 } & \text { KJSJ } \\ \text { Percentage } & \text { KJSK } \\ & \\ 90 \text { and over } & \text { KJSL } \\ \text { Percentage } & \text { KJSM } \\ & \\ \text { Unknown age } & \text { EW3Y } \\ \text { Percentage } & \text { EW3Z } \\ & \end{array}$

Total all ages

KJSA

$\begin{array}{rr}1364.10 & 1341.50 \\ 31.40 & 30.60 \\ 1150.00 & 1160.10 \\ 26.50 & 26.50 \\ 887.10 & 903.00 \\ 20.40 & 20.60 \\ 593.30 & 596.90 \\ 13.70 & 13.60 \\ 246.40 & 273.10 \\ 5.70 & 6.20 \\ 100.20 & 103.60 \\ 2.30 & 2.40 \\ 1.10 & 1.20 \\ - & -\end{array}$

1332.77
30.03
1177.96
26.54
918.47
20.70
604.74
13.63
296.36
6.68

1350.61
29.84

1389.85 29.99

JSE

KJSF

KJSH

JSJ

KJSL

KJSM

106.13

2.39

1.45

4379.50

4437.99

1205.70

1232.97

26.61

932.17

942.03

20.33

$\begin{array}{rr}614.77 & 627.28 \\ 13.58 & 13.54\end{array}$

$317.90 \quad 335.49$

$\begin{array}{ll}7.02 & 7.24\end{array}$

$105.33 \quad 105.62$

$\begin{array}{rr}2.33 & 2.28\end{array}$

$0.19 \quad 0.24$

4342.20

4526.79

4633.62

\section{Women:}

$\begin{array}{ll}\text { Age-groups: } & \\ 60-64 & \text { KJSO } \\ \text { Percentage } & \text { KJSP } \\ \text { 65-69 } & \text { KJSQ } \\ \text { Percentage } & \text { KJSR } \\ 70-74 & \text { KJSS } \\ \text { Percentage } & \text { KJST } \\ \text { 75-79 } & \text { KJSU } \\ \text { Percentage } & \text { KJSV } \\ \text { 80-84 } & \text { KJSW } \\ \text { Percentage } & \text { KJSX } \\ \text { 85-89 } & \text { KJSY } \\ \text { Percentage } & \text { KJSZ } \\ \text { 90 and over } & \text { KJTA } \\ \text { Percentage } & \text { KJTB } \\ \text { Unknown age } & \text { EW42 } \\ \text { Percentage } & \text { EW43 } \\ \text { Total all ages } & \text { KJSN }\end{array}$

1498.70

20.80

1464.20

20.30

1314.50

18.20

1158.60

16.10

951.60

13.20

511.00

7.10
314.90

4.40

1.30

7214.70

1 See chapter text.

2 Caseloads include both contributory and non-contributory state pensioners.

\begin{tabular}{rrrr}
1524.00 & 1628.19 & 1695.88 & 1734.92 \\
21.00 & 21.98 & 22.47 & 22.62 \\
1453.10 & 1456.08 & 1484.80 & 1527.47 \\
20.00 & 19.65 & 19.67 & 19.91 \\
& & & 1366.91 \\
1312.70 & 1322.14 & 1343.22 & 17.82 \\
18.10 & 17.85 & 17.80 & 166.20 \\
1165.50 & 1168.86 & 1170.01 & 15.20 \\
16.00 & 15.78 & 15.50 & 921.01 \\
933.30 & 923.70 & 919.11 & 12.01 \\
12.90 & 12.47 & 12.18 & 643.50 \\
552.70 & 587.91 & 621.15 & 8.39 \\
7.60 & 7.94 & 8.23 & 310.07 \\
319.40 & 319.90 & 313.66 & 4.04 \\
4.40 & 4.32 & 4.16 & 0.38 \\
1.50 & 1.67 & 0.37 &.. \\
- & - &.. & 7670.44 \\
7262.30 & 7408.44 & 7548.20 & 15 \\
\hline
\end{tabular}

Sources: Department for Work and Pensions; Work and Pensions Longitudinal Study (WPLS); 
War pensions: estimated number of pensioners ${ }^{1}$

Great Britain

At 31 March each year

Thousands

\begin{tabular}{|c|c|c|c|c|c|c|c|c|c|c|c|c|}
\hline & & 1999 & 2000 & 2001 & 2002 & 2003 & 2004 & 2005 & $2006^{2}$ & 2007 & 2008 & 2009 \\
\hline Disablement & $\mathrm{KADH}$ & 248.93 & 240.76 & 231.62 & 221.80 & 212.18 & 201.55 & 191.75 & 182.80 & 173.85 & 165.17 & 157.13 \\
\hline Widows and dependants & KADI & 55.85 & 54.92 & 52.71 & 50.98 & 48.61 & 46.04 & 43.55 & 41.05 & 38.69 & 36.10 & 33.6 \\
\hline Total & KADG & 306.06 & 295.67 & 284.33 & 272.78 & 260.79 & 247.59 & 235.30 & 223.85 & 212.54 & 201.27 & 190.75 \\
\hline
\end{tabular}

1 See chapter text. From 1914 war, 1939 war and later service.

Source: Ministry of Defence/DASA (Health Information): 01225467801

2 The discontinuity between 2005 and 2006 is due to improvements in data

processing.

\subsection{6 income support't by ystatistical froup? : number of claimants receiving weekly payment}

Great Britain

Thousands ${ }^{4}$

\begin{tabular}{|c|c|c|c|c|c|c|c|}
\hline & & 2004 & 2005 & 2006 & 2007 & 2008 & 2009 \\
\hline All income support claimants ${ }^{5}$ & F8YY & 2192.6 & 2139.8 & 2114.8 & 2117.7 & 2091.5 & 1979.8 \\
\hline Incapacity Benefits & F8YZ & 1205.2 & 1193.8 & 1183.2 & 1184.7 & 1182.5 & 1088.5 \\
\hline Lone Parent & F8z2 & 823.3 & 789.3 & 774.9 & 765.6 & 738.6 & 720.5 \\
\hline Carer & F8z3 & 78.40 & 79.00 & 80.20 & 82.80 & 85.70 & 92.10 \\
\hline Others on Income Related Benefits & F8Z4 & 85.90 & 77.70 & 76.50 & 84.60 & 84.70 & 78.70 \\
\hline
\end{tabular}

1 Figures are given at May each year and are taken from the DWP 100\%

Sources: Department for Work and Pensions; Work and Pensions Longitudinal Study (WPLS).

2 From 27th October 2008, new claims to Income Support can also be allo-

cated, on incapacity grounds, to the newly introduced Employment and

Support Allowance (ESA).

3 Statistical groups are defined as follows:

Incapacity Benefits- claimants aged under 60 on Incapacity Benefit or Se-

vere Disablement Allowance;

Lone Parent - single claimants aged under 60 with dependants not in re-

ceipt of IB/SDA;

Carer- claimants aged under 60 entitled to Carer's Allowance;

Other Income Related Benefit- claimants not in one of the above cate-

gories.

4 Figures are rounded to the nearest hundred and quoted in thousands.

5 Totals may not sum due to rounding.

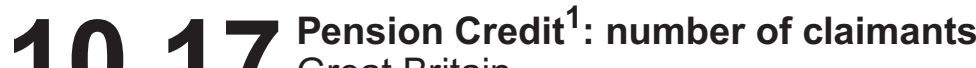 Great Britain \\ End of May}

\section{All Pension Credit
Guarantee Credit Only
Guarantee Credit Only and}

Savings Credit

1 Source: DWP 100\% Work and Pensions Longitudinal study (WPLS).

2 All figures are rounded to the nearest hundred and expressed in thousands.

2004

\section{F8z5}

2490.8

735.0
1269.5

486.0

\begin{tabular}{lr} 
F8Z5 & 2490.8 \\
& \\
F8Z6 & 735.0 \\
F8Z7 & 1269.5 \\
F8z8 & 486.0 \\
\hline
\end{tabular}

200
26
7
13
5
5

2005

2006

$\begin{array}{r}767.3 \\ 1321.7 \\ 593.7 \\ \hline\end{array}$

2717.4

$\begin{array}{r}775.6 \\ 1343.2 \\ 598.6 \\ \hline\end{array}$

\begin{tabular}{rrr} 
& & Thousands $^{2}$ \\
\hline 2007 & 2008 & 2009 \\
\hline 2733.5 & 2719.1 & 2730.6 \\
& & \\
805.7 & 882.1 & 925.7 \\
1330.1 & 1246.2 & 1205.2 \\
597.7 & 590.8 & 599.6 \\
\hline
\end{tabular}

Sources: Department for Work and Pensions; Information Directorate 


\section{Income support: average weekly amounts of benefit ${ }^{1,2,3}$ \\ - O Great Britain}

\begin{tabular}{|c|c|c|c|c|c|c|c|}
\hline ת ats & & 2004 & 2005 & 2006 & 2007 & 2008 & 2009 \\
\hline All income support claimants & $\mathrm{F} 8 \mathrm{ZF}$ & 91.14 & 85.81 & 83.41 & 82.29 & 82.35 & 84.94 \\
\hline
\end{tabular}

1 Figures are given at May each year and are taken from the DWP $100 \%$ Work and Pensions Longitudinal Study (WPLS).

Sources: Department for Work and Pensions;

2 From 27th October 2008, new claims to Income Support can also be allo-

cated, on incapacity grounds, to the newly introduced Employment and

Support Allowance (ESA).

3 Average amounts are rounded to the nearest penny.

4 Statistical groups are defined as follows:

Incapacity Benefits- claimants under 60 on incapacity benefit or Severe Disablement Allowance;

Lone Parent- single claimants aged under 60 with dependants not in receipt of IB/SDA;

Carer- claimants aged under 60 entitled to Carer's Allowance;

Other Income Related Benefit- claimants not in one of the above categories.

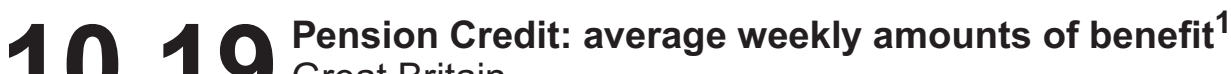 Great Britain}

As at May

\begin{tabular}{|c|c|c|c|c|c|c|c|}
\hline & & 2004 & 2005 & 2006 & 2007 & 2008 & 2009 \\
\hline All Pension Credit & F8ZA & 42.30 & 43.62 & 46.75 & 50.04 & 52.69 & 55.56 \\
\hline Guarantee Credit Only & F8ZB & 71.91 & 75.43 & 79.56 & 83.74 & 85.07 & 88.86 \\
\hline Guarantee Credit and Savings Credit & F8ZC & 37.51 & 39.87 & 43.11 & 46.11 & 48.29 & 50.81 \\
\hline Savings Credit only & F8ZD & 10.03 & 10.83 & 12.39 & 13.36 & 13.62 & 13.71 \\
\hline
\end{tabular}

1 Figures are given in each May and are taken from the DWP $100 \%$ Work and Pensions Longitudinal Study (WPLS).

Sources: Department for Work and Pensions;

2 Average amounts are shown as pounds per week and rounded to the near-

\section{Summary of government expenditure on social services and housing 1 Years ended 31 March}

\begin{tabular}{|c|c|c|c|c|c|c|c|c|c|}
\hline & & & & & & & & & $£$ million \\
\hline & & $\begin{array}{r}2001 \\
102\end{array}$ & $\begin{array}{r}2002 \\
103\end{array}$ & $\begin{array}{r}2003 \\
104\end{array}$ & $\begin{array}{r}2004 \\
105\end{array}$ & $\begin{array}{r}2005 \\
106\end{array}$ & $\begin{array}{r}2006 \\
107\end{array}$ & $\begin{array}{r}2007 \\
108\end{array}$ & $\begin{array}{r}2008 \\
109\end{array}$ \\
\hline \multicolumn{10}{|l|}{ Final Consumption Expenditure } \\
\hline Education & QYWZ & 53779 & 58505 & 63866 & 69216 & 75368 & 78830 & 82564 & 89018 \\
\hline Health & QYXA & 62263 & 68794 & 75844 & 83637 & 89998 & 95980 & 103512 & 110435 \\
\hline Personal social services & GB7F & 15033 & 17232 & 19927 & 21906 & 23413 & 24315 & 25354 & 26908 \\
\hline Social benefits & GG50 & 121098 & 126345 & 135591 & 142004 & 145268 & 149793 & 159832 & 173476 \\
\hline Housing & QYXD & 8043 & 9276 & 11768 & 14527 & 14391 & 16055 & 18396 & 17734 \\
\hline Total government expenditure & $\mathrm{GH} 2 \mathrm{~K}$ & 260216 & 280152 & 306996 & 331290 & 348438 & 364973 & 389658 & 417571 \\
\hline
\end{tabular}

Total government expenditure on social services and housing as a percentage of GDP

GGN7

25.2

25.7

26.6

$\begin{array}{lllll}27.2 & 27.4 & 27.2 & 27.5 & 29.2\end{array}$

1 See chapter text. 
Social protection

$£$ million

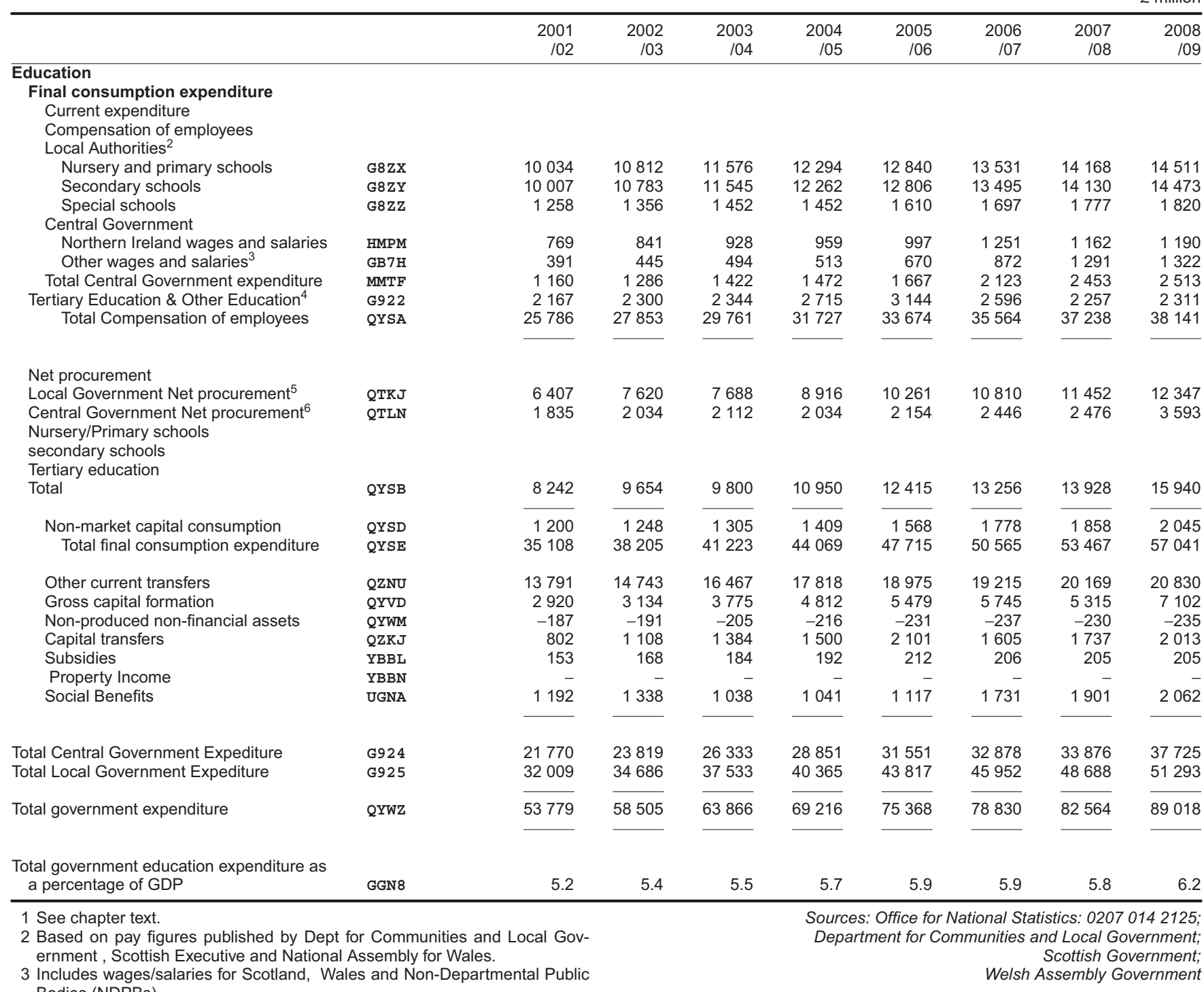

Bodies (NDPBs)

4 Includes Higher, Further, Adult and Continuing education

5 Net of VAT.

6 Includes Central Government Net Procurement on NDPBs, Scotland,

Wales, Northern Ireland and Education in Healthcare. 
Summary of Government expenditure on Health ${ }^{1}$

Years ended 31 March

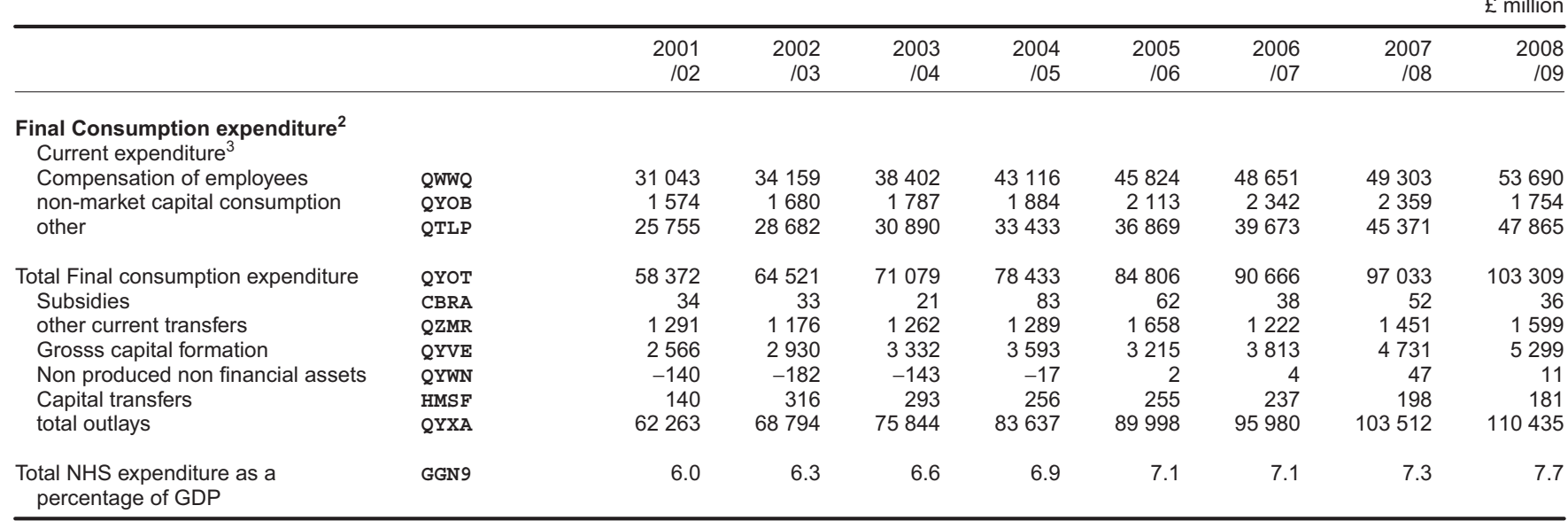

1 See chapter text.

2 Figures are based on Departmental Expenditure reported to HM Treasury

Source: Office for National Statistics: 02070142125

Statistics database.

3 Includes expenditure by Dept. of Health, NHS Trusts, Scottish Government,

Welsh Assembly Government and Northern Ireland Executive.

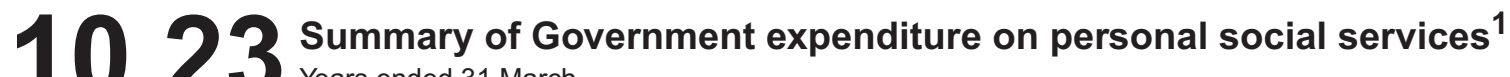 \\ a Years ended 31 March}

\begin{tabular}{|c|c|c|c|c|c|c|c|c|c|}
\hline & & $\begin{array}{r}2001 \\
/ 02\end{array}$ & $\begin{array}{r}2002 \\
103\end{array}$ & $\begin{array}{r}2003 \\
104\end{array}$ & $\begin{array}{r}2004 \\
105\end{array}$ & $\begin{array}{r}2005 \\
106\end{array}$ & $\begin{array}{r}2006 \\
107\end{array}$ & $\begin{array}{r}2007 \\
108\end{array}$ & $\begin{array}{r}2008 \\
109\end{array}$ \\
\hline \multicolumn{10}{|l|}{ Personal social services } \\
\hline Total & GB7D & 674 & 908 & 951 & 1149 & 1153 & 1085 & 1131 & 1200 \\
\hline $\begin{array}{l}\text { Local Authorities Current Expenditu } \\
\text { Compensation of employees } \\
\text { Net Procurement }\end{array}$ & $\begin{array}{l}\text { CFCR } \\
\text { QWSB }\end{array}$ & $\begin{array}{l}5936 \\
8349\end{array}$ & $\begin{array}{l}6385 \\
9859\end{array}$ & $\begin{array}{r}6940 \\
11951\end{array}$ & $\begin{array}{r}7449 \\
13207\end{array}$ & $\begin{array}{r}7889 \\
14250\end{array}$ & $\begin{array}{r}8230 \\
14864\end{array}$ & $\begin{array}{r}8436 \\
15637\end{array}$ & $\begin{array}{r}8938 \\
16610\end{array}$ \\
\hline Total & GB7E & 14285 & 16244 & 18891 & 20656 & 22139 & 23094 & 24073 & 25548 \\
\hline Capital Expenditure & GDZU & 74 & 80 & 85 & 101 & 121 & 136 & 150 & 160 \\
\hline Total Final Consumption Expenditure & GB7F & 15033 & 17232 & 19927 & 21906 & 23413 & 24315 & 25354 & 26908 \\
\hline $\begin{array}{l}\text { Total government expenditure as } \\
\text { a percentage of GDP }\end{array}$ & $\mathrm{GGO} 2$ & 1.5 & 1.6 & 1.8 & 1.8 & 1.9 & 1.8 & 1.8 & 1.9 \\
\hline
\end{tabular}


Social protection

Summary of Government expenditure on social security benefits ${ }^{1}$ and administration

Years ended 31 March

$£$ million

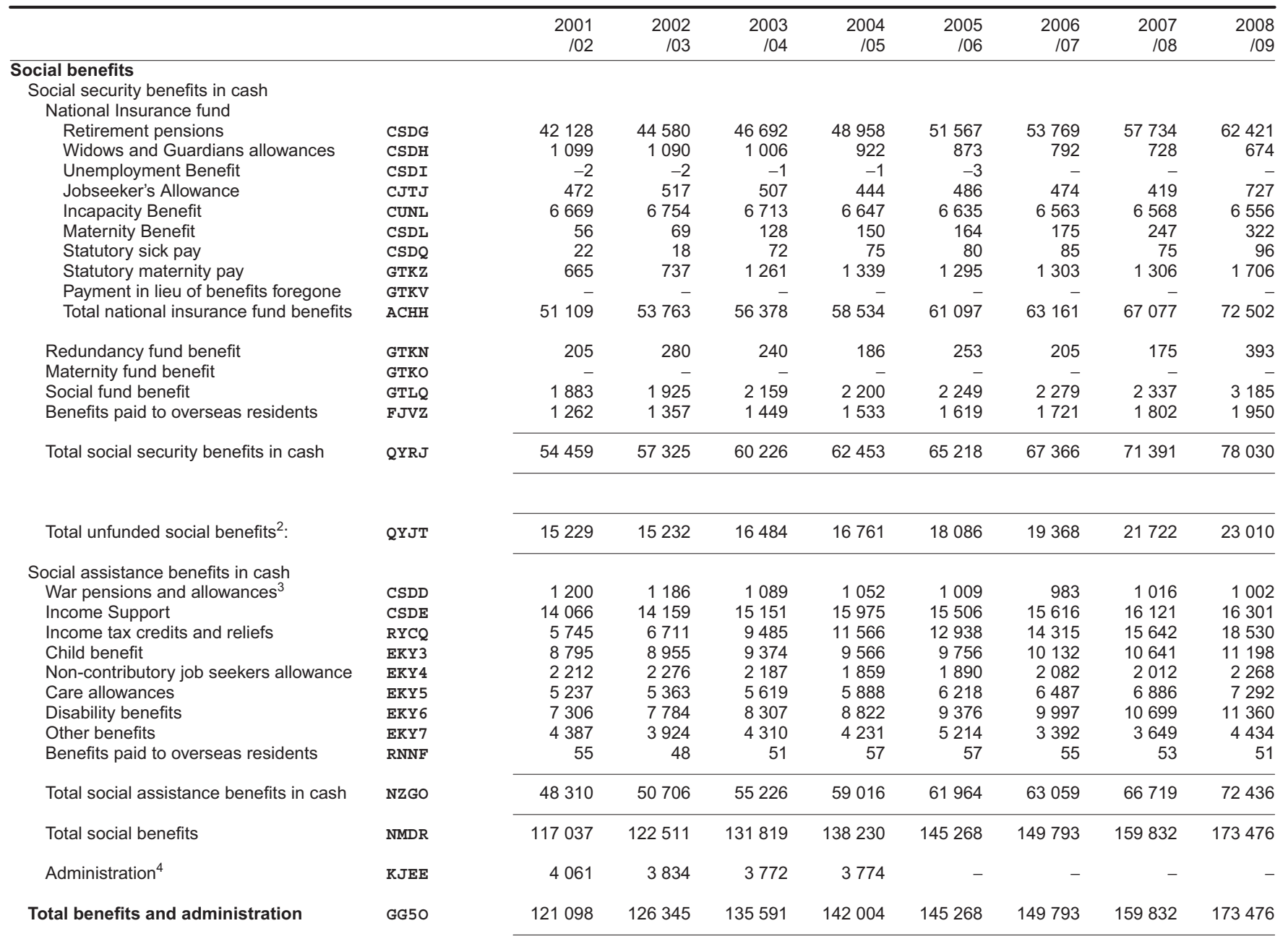

Total government benefit expenditure as a percentage of GDP

GGO3

12.2

12.2

11.7

11.7

11.4

11.1

11.3

12.1

See chapter text. Figures are based on table 5.2.4s of the Blue Book 2007.

They are not fully comparable with earlier editions of the Annual Abstract.

Sources: Office for National Statistics: 02070142125 ; Department for Work and Pensions

\& NHS inflationary pensions increase payments.

3 From 2002/03 War Pensions are administered by the Ministry of Defence.

4 Figures published by HM Treasury in Public Expenditure Statistical Analy-

ses. A separate figure for administration is no longer published. 


\section{Social protection}

\section{Summary of Government expenditure on housing ${ }^{1}$ \\ - Years ended 31 March}

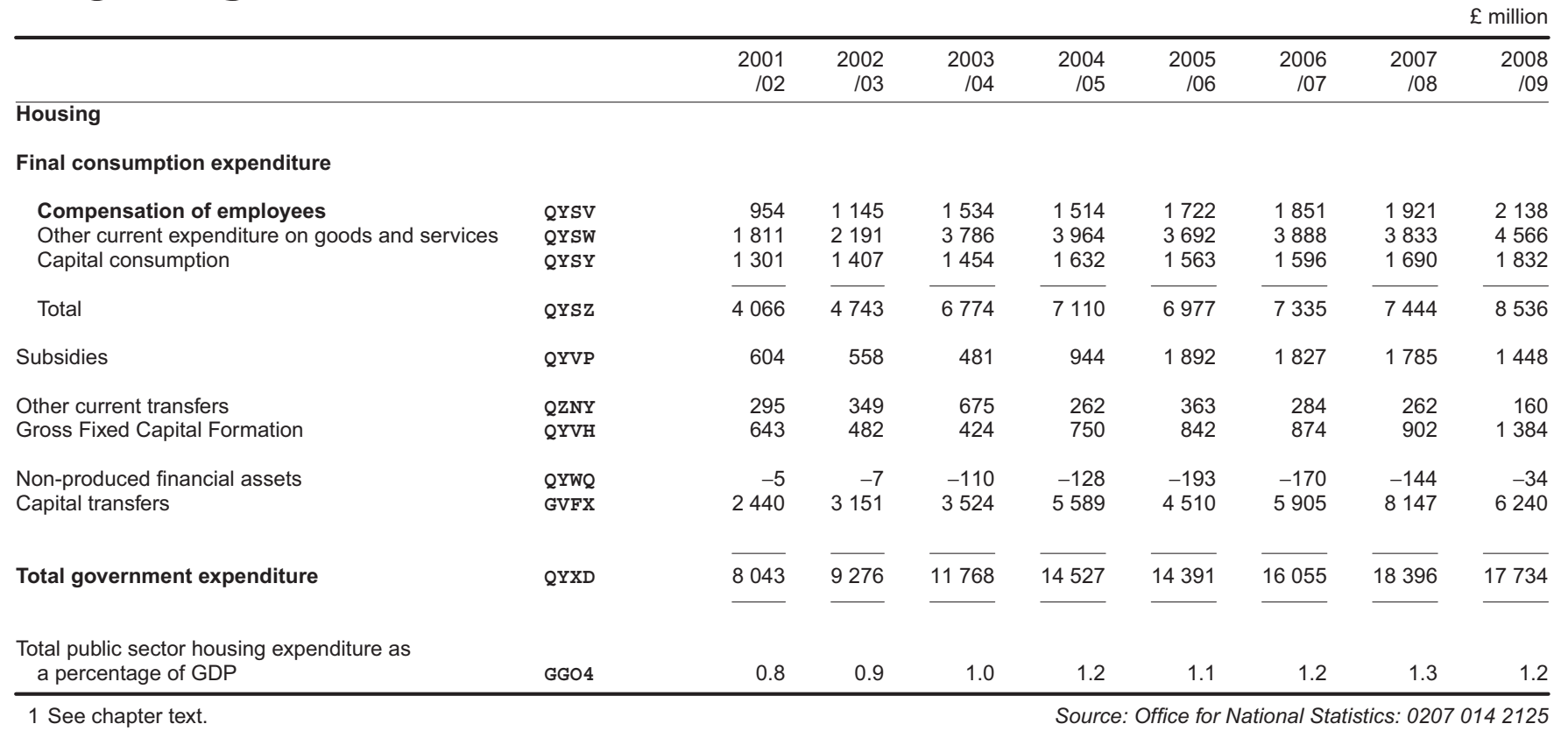

